\title{
Islatravir Is Not Expected to Be a Victim or Perpetrator of Drug-Drug Interactions via Major Drug-Metabolizing Enzymes or Transporters
}

\author{
Kelly Bleasby, Robert Houle, Michael Hafey, Meihong Lin, Jingjing Guo, Bing Lu, Rosa I. Sanchez \\ and Kerry L. Fillgrove*
}

check for updates

Citation: Bleasby, K.; Houle, R.; Hafey, M.; Lin, M.; Guo, J.; Lu, B.; Sanchez, R.I.; Fillgrove, K.L. Islatravir Is Not Expected to Be a Victim or Perpetrator of Drug-Drug Interactions via Major Drug-Metabolizing Enzymes or Transporters. Viruses 2021, 13, 1566. https://doi.org/10.3390/v13081566

Academic Editor: Erik De Clercq

Received: 25 May 2021

Accepted: 23 July 2021

Published: 7 August 2021

Publisher's Note: MDPI stays neutral with regard to jurisdictional claims in published maps and institutional affiliations.

Copyright: (c) 2021 by the authors. Licensee MDPI, Basel, Switzerland. This article is an open access article distributed under the terms and conditions of the Creative Commons Attribution (CC BY) license (https:// creativecommons.org/licenses/by/ $4.0 /)$.
Merck \& Co., Inc., Kenilworth, NJ 07033, USA; Kelly_Bleasby@merck.com (K.B.); robert_houle@merck.com (R.H.); michael_hafey@merck.com (M.H.); meihong.lin@merck.com (M.L.); jingjing.guo@merck.com (J.G.); bing_lu@merck.com (B.L.); rosa_sanchez@merck.com (R.I.S.)

* Correspondence: Kerry_fillgrove@merck.com; Tel.: +1-215-652-6139

\begin{abstract}
Islatravir (MK-8591) is a nucleoside reverse transcriptase translocation inhibitor in development for the treatment and prevention of HIV-1. The potential for islatravir to interact with commonly co-prescribed medications was studied in vitro. Elimination of islatravir is expected to be balanced between adenosine deaminase-mediated metabolism and renal excretion. Islatravir did not inhibit uridine diphosphate glucuronosyltransferase 1A1 or cytochrome p450 (CYP) enzymes CYP1A2, 2B6, 2C8, 2C9, 2C19, 2D6, or 3A4, nor did it induce CYP1A2, 2B6, or 3A4. Islatravir did not inhibit hepatic transporters organic anion transporting polypeptide (OATP) 1B1, OATP1B3, organic cation transporter (OCT) 1, bile salt export pump (BSEP), multidrug resistance-associated protein (MRP) 2, MRP3, or MRP4. Islatravir was neither a substrate nor a significant inhibitor of renal transporters organic anion transporter (OAT) 1, OAT3, OCT2, multidrug and toxin extrusion protein (MATE) 1, or MATE2K. Islatravir did not significantly inhibit P-glycoprotein and breast cancer resistance protein (BCRP); however, it was a substrate of BCRP, which is not expected to be of clinical significance. These findings suggest islatravir is unlikely to be the victim or perpetrator of drug-drug interactions with commonly co-prescribed medications, including statins, diuretics, anti-diabetic drugs, proton pump inhibitors, anticoagulants, benzodiazepines, and selective serotonin reuptake inhibitors.
\end{abstract}

Keywords: 4'-ethynyl-2-fluoro-2'-deoxyadenosine (EFdA); islatravir; MK-8591; cytochrome p450; drug-drug interaction; drug transporters; HIV-1; nucleoside reverse transcriptase translocation inhibitor; concomitant medication; antiretroviral agents

\section{Introduction}

HIV-1 can now be managed as a chronic illness with lifelong combination antiretroviral therapy (ART); the life expectancy of people living with HIV (PLWH) is approaching that of people without HIV, particularly in high-income nations [1,2]. Although progress in the treatment of HIV-1 with ART has greatly reduced the morbidity and mortality associated with this infection, substantial disease management challenges remain [2-4].

PLWH have an increased risk of accelerated aging and the development of comorbidities [5,6], including diabetes, cardiovascular disease, chronic liver disease, and chronic kidney disease $[2,7,8]$. Therefore, in addition to ART, PLWH often require medications to treat their comorbidities, such as statins, diuretics, antidiabetic drugs, or benzodiazepines, which can lead to considerable polypharmacy and necessitates consideration of potential drug-drug interactions, adverse events, food restrictions, and complicated administration schedules [9-11]. The high frequency of drug interactions seen in PLWH receiving polypharmacy can result in adverse health outcomes and has typically required treatment modification or increased monitoring [12]. 
Pharmacokinetic drug interactions result from changes in plasma concentrations of a 'victim' drug caused by a 'perpetrator' drug altering the metabolism or transportermediated disposition of the victim drug [13]. An increase in victim drug concentrations usually occurs when metabolism or transporter-dependent elimination of that drug is inhibited by a perpetrator, increasing the risk for accumulation in plasma and tissues, as well as drug-related toxicities. Conversely, when metabolism or transporter-dependent elimination of the victim drug is augmented by the perpetrator drug, concentrations of the victim drug will decrease, which may reduce its efficacy. For antiretroviral agents, the result is suboptimal suppression of HIV, leading to the development of resistance, viral rebound, and increased risk of virus transmission. Characterization of the potential for drug interactions between new antiretroviral agents and established antiretroviral agents with which they may be co-administered, or with common non-HIV medications, is currently stipulated in regulatory agency guidance [14-16].

Islatravir (MK-8591) is a nucleoside reverse transcriptase translocation inhibitor (NRTTI) in development for the treatment and prevention of HIV-1 (Figure 1A) [17,18]. Islatravir inhibits reverse transcriptase (RT) by multiple mechanisms of action, including RT translocation inhibition and delayed chain termination through viral DNA structural changes [19-21]. Islatravir is being developed to address the need for new antiretroviral agents with favorable safety and tolerability profiles, high potency, and a high barrier to the development of resistance that may also allow for simplification of treatment [22].

A<smiles>C#C[C@]1(CO)O[C@@H](n2cnc3c(N)nc(F)nc32)C[C@@H]1O</smiles>

B

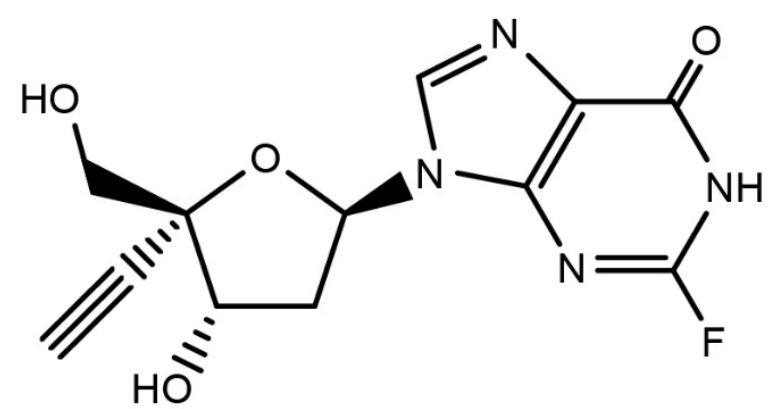

Figure 1. Structure of (A) islatravir and (B) metabolite M4 4'-ethynyl-2-fluoro-2'-deoxyinosine.

Islatravir has a favorable pharmacokinetic profile and is rapidly converted intracellularly by endogenous kinases to its active triphosphate (TP), islatravir-TP, which inhibits RT by multiple mechanisms to suppress HIV-1 replication [18,20,21,23-25]. In treatment-naive $\mathrm{PLWH}$, islatravir was rapidly absorbed and plasma exposure was approximately dose proportional after oral administration with similar pharmacokinetics (PK) in adults without HIV. Islatravir-TP had a long intracellular half-life of $~ 78.5-128 \mathrm{~h}$, in agreement with the viral load reduction maintained for 7 days after a single administration of islatravir at a dose as low as $0.5 \mathrm{mg}$ [26].

In treatment-naïve PLWH, islatravir administered orally in daily doses of between 0.5 and $30 \mathrm{mg}$ effectively suppressed viral load for at least 7 days [26]. Islatravir was gener- 
ally well tolerated in participants with and without HIV across a range of doses [26,27]. Owing to the high potency, high barrier to the development of resistance, and long intracellular half-life of islatravir-TP, islatravir has the potential to be effective in a variety of dosing options and regimens for the treatment and prevention of HIV-1. The combination of islatravir with doravirine, a non-nucleoside reverse transcriptase inhibitor (NNRTI), is currently being evaluated in a comprehensive phase 3 clinical program across diverse groups of PLWH, including treatment-naive and treatment-experienced populations (ClinicalTrials.gov ID: NCT04223778, https:/ / clinicaltrials.gov/ct2/show /NCT042 23778; NCT04223791, https: / / clinicaltrials.gov/ct2/ show / NCT04223791; NCT04233879, https:/ / clinicaltrials.gov/ct2/show/NCT04233879, accessed on 22 July 2021). In heavily treatment experienced PLWH who are failing their current ART regimen, islatravir and doravirine will be administered in combination with optimized background ART (ClinicalTrials.gov (accessed on 22 July 2021) ID: NCT04233216). Islatravir is also being investigated for HIV-1 pre-exposure prophylaxis in at-risk individuals as a once-monthly oral regimen and a once-yearly implant [28,29].

Herein we describe in vitro studies conducted to characterize the distribution and metabolism of islatravir and to establish the potential for islatravir to interact with other drugs via major drug-metabolizing enzymes, and with transporters involved in clinically relevant drug interactions. The selection of drug-metabolizing enzymes and transporters in these studies was based upon the known metabolic properties and elimination pathways for islatravir and commonly prescribed concomitant medications in PLWH, aligning with regulatory guidance on the evaluation of the drug interaction potential of new molecular entities $[14,16,30]$. The selected drug-metabolizing enzymes and transporters involved in clinically relevant drug interactions include cytochrome P450 (CYP) enzymes and uridine diphosphate glucuronosyltransferase 1A1 (UGT1A1); the hepatic uptake transporters organic anion transporting polypeptide (OATP) 1B1, OATP1B3, and organic cation transporter (OCT) 1; the renal uptake transporters organic anion transporter (OAT)1, OAT3, and OCT2; the efflux transporters multidrug and toxin extrusion protein (MATE) 1 and MATE2K; and the widely expressed efflux transporters multidrug resistance protein 1 P-glycoprotein (MDR1 P-gp) and breast cancer resistance protein (BCRP) [14-16,31,32]. The bile salt export pump (BSEP) and multidrug resistance-associated protein (MRP) 2, MRP3, and MRP4 were also investigated due to their association with drug-induced liver injury [33]. The locations of these selected drug-metabolizing enzymes and transporters of clinical interest, along with the main elimination pathways of commonly prescribed medications in PLWH, are illustrated in Figure 2. 
A

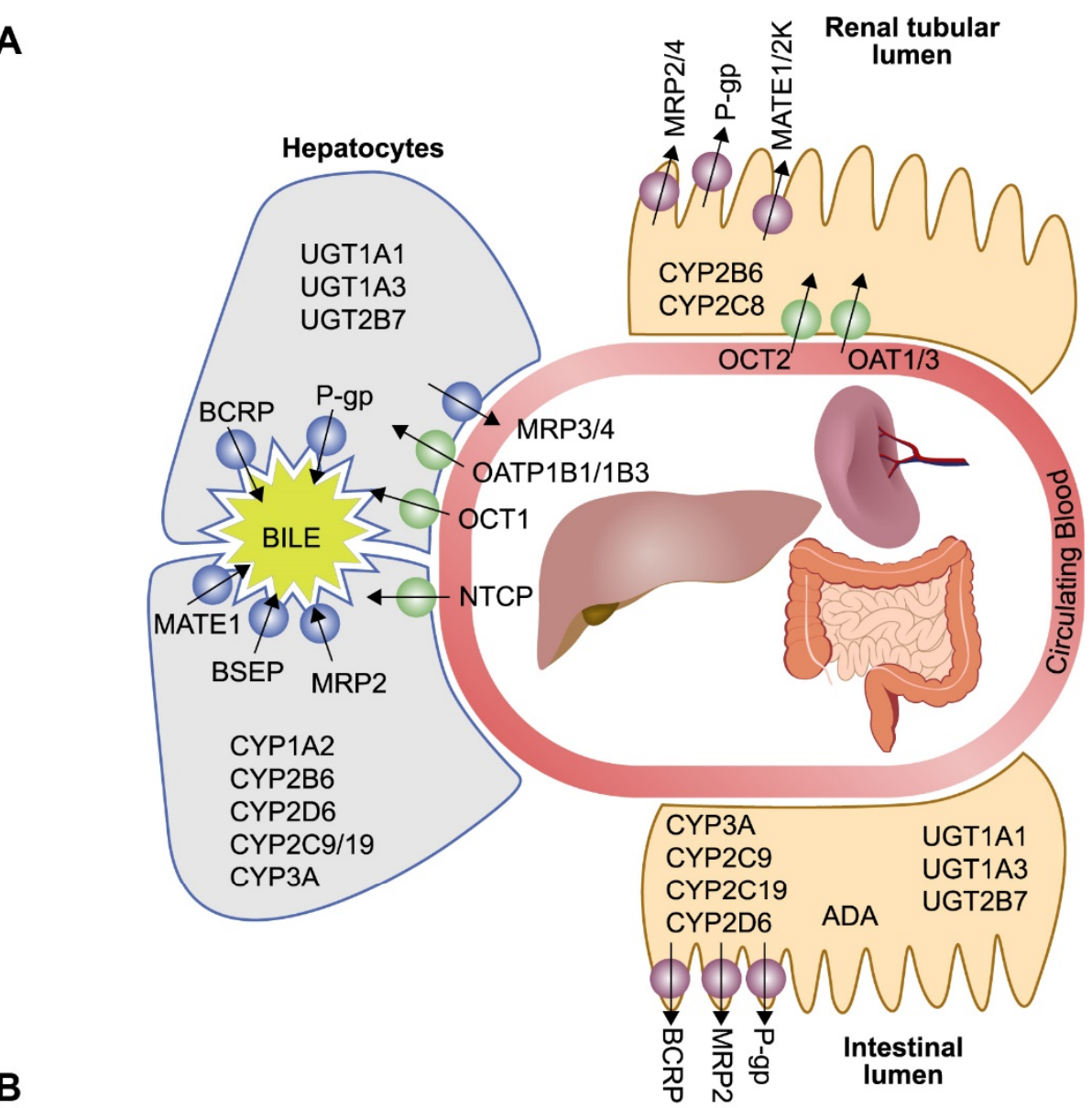

B

\begin{tabular}{|c|c|}
\hline $\begin{array}{l}\text { Co-administered } \\
\text { medication }\end{array}$ & Major distribution and elimination pathways \\
\hline Atorvastatin & $\begin{array}{l}\text { Extensive metabolism by CYP3A4 and elimination primarily in bile with active } \\
\text { hepatic uptake via OATP1B1 and efflux via MDR1 P-gp and BCRP }\end{array}$ \\
\hline Rosuvastatin & $\begin{array}{l}\text { Intestinal absorption modulated by BCRP efflux. Elimination mainly unchanged in } \\
\text { urine (active renal secretion mediated via OAT3) and feces (biliary excretion via } \\
\text { OATP1B1, NTCP, and OATP1B3) }\end{array}$ \\
\hline Omeprazole & Extensive metabolism by CYP2C19, and to a lesser extent by CYP3A4 \\
\hline Metformin & $\begin{array}{l}\text { Elimination mainly unchanged in urine with active renal secretion mediated via } \\
\text { OCT2, MATE1, and MATE2K. Distribution into liver (site of action) via OCT1 }\end{array}$ \\
\hline $\begin{array}{l}\text { Suboxone } \\
\text { (buprenorphine } \\
\text { \& naloxone) }\end{array}$ & $\begin{array}{l}\text { Buprenorphine: elimination primarily via CYP3A metabolism, with glucuronidation } \\
\text { by UGT1A1, UGT1A3, and UGT2B7 } \\
\text { Naloxone: elimination primarily via UGT2B7 metabolism }\end{array}$ \\
\hline Clopidogrel & $\begin{array}{l}\text { Elimination primarily via metabolism by carboxylase to form the inactive carboxylic } \\
\text { acid and by primarily CYP2C19 with contributions from CYP1A2, CYP2B6, } \\
\text { CYP2C9, and CYP3A to form the active metabolite }\end{array}$ \\
\hline Alprazolam & $\begin{array}{l}\text { Elimination primarily via CYP3A metabolism with excretion of unchanged drug } \\
\text { a minor pathway }\end{array}$ \\
\hline Citalopram & $\begin{array}{l}\text { Elimination primarily via CYP2C19 and CYP2D6 metabolism with contribution } \\
\text { from CYP3A4 }\end{array}$ \\
\hline
\end{tabular}

Figure 2. Key elimination pathways of commonly co-prescribed medications * [34-53]. (A) Location of drug-metabolizing enzymes and transporters of interest. (B) Elimination and distribution pathways for co-administered medications with islatravir *. ADA, adenosine deaminase; BCRP, breast cancer resistance protein; BSEP, bile salt export pump; CYP, cytochrome P450; MATE, multidrug and toxin extrusion protein; MDR1 P-gp, multidrug resistance protein 1 P-glycoprotein; MRP, multidrug resistance-associated protein; $\mathrm{OAT}$, organic anion transporter; OATP, organic anion transporting polypeptide; $\mathrm{OCT}$, organic cation transporter; $\mathrm{NTCP}$, sodium taurocholate co-transporting polypeptide; UGT, uridine diphosphate glucuronosyltransferase. ${ }^{*}$ Commonly prescribed co-medications taken from European Medicines Agency scientific advice on metabolic and elimination pathways for key medications expected to be taken concomitantly with islatravir. 


\section{Materials and Methods}

\subsection{Islatravir Distribution in Plasma}

Islatravir plasma protein binding was determined as previously described by equilibrium dialysis [54]. Briefly, $0.1,1$, and $10 \mu \mathrm{M}$ islatravir was added to human, mouse, rat, rabbit, or monkey plasma and dialyzed against an equal volume of phosphate-buffered saline ( $\mathrm{pH} 7.4$ ) at $37^{\circ} \mathrm{C}$ under $10 \% \mathrm{CO}_{2}$, for $24 \mathrm{~h}$. Samples were extracted with the addition of acetonitrile, vortex-mixed, and centrifuged. The resulting supernatants were analyzed by liquid chromatography with tandem mass spectrometry (LC-MS/MS). The unbound fraction in plasma was calculated as Fraction unbound $=$ islatravir concentration in buffer chamber/islatravir concentration in plasma chamber.

Distribution of islatravir between red blood cells and plasma in human blood was determined at select concentrations ranging from 0.01 to $10 \mu \mathrm{M}$. Islatravir was added to aliquots of blood and incubated under $5 \% \mathrm{CO}_{2}$ for $60 \mathrm{~min}$ at $37^{\circ} \mathrm{C}$, followed by separation of the red blood cells from the plasma via centrifugation. To assess its initial whole blood concentration, islatravir was added to aliquots of plasma and incubated under $5 \% \mathrm{CO}_{2}$ for $60 \mathrm{~min}$ at $37^{\circ} \mathrm{C}$ to serve as a surrogate for whole blood. Samples were extracted with the addition of acetonitrile, vortex-mixed, and centrifuged. The resulting supernatants were analyzed by LC-MS/MS. The blood to plasma ratio (B:P) was calculated as B:P = islatravir concentration in whole blood/islatravir concentration in plasma separated from blood.

\subsection{Characterization of Islatravir Metabolism in Intestinal S9 and Metabolism by Human Adenosine Deaminase}

The metabolism of islatravir was studied in human intestinal S9 fraction (Xenotech, LLC [Kansas City, KS, USA]). [ ${ }^{3} \mathrm{H}$ ]islatravir $(5 \mu \mathrm{M})$ was incubated at $37^{\circ} \mathrm{C}$ for $3 \mathrm{~h}$ in $0.1 \mathrm{M}$ potassium phosphate buffer ( $\mathrm{pH} 7.4$ ) containing $1.0 \mathrm{mg} / \mathrm{mL}$ S9 protein, $5 \mathrm{mM}$ magnesium chloride, and $1 \mathrm{mM}$ NADPH. Reactions were terminated with a stop solution containing $6 \mathrm{mM}$ EDTA and $6 \mathrm{mM}$ EGTA in $70 \%$ methanol. Samples were vortex mixed, centrifuged, and the supernatants were subjected to radiometric LC-MS/MS analysis.

The metabolism of islatravir was also evaluated with recombinant human adenosine deaminase (ADA). Islatravir $(50 \mu \mathrm{M})$ was incubated at $37{ }^{\circ} \mathrm{C}$ for $3 \mathrm{~h}$ in $0.05 \mathrm{M}$ HEPES buffer ( $\mathrm{pH}$ 7.4) containing 1 unit/mL of recombinant human ADA (Novus Biologicals, LLC [Centennial, CO, USA]). Reactions were terminated by the addition of acetonitrile, and the samples were vortex-mixed and centrifuged, and the supernatants were subjected to LC-MS/MS analysis.

Enzyme kinetics were evaluated using increasing concentrations of islatravir incubated with recombinant human ADA, pre-incubated in potassium phosphate buffer for $10 \mathrm{~min}$ at $37^{\circ} \mathrm{C}$. Reactions were initiated by the addition of islatravir for $15 \mathrm{~min}$ and terminated by acetonitrile:methanol containing stable isotope-labeled islatravir $\left(\left[{ }^{13} \mathrm{C}^{15} \mathrm{~N}_{3}\right] \mathrm{ISL}\right)$. Samples were then vortex-mixed and centrifuged, and the resulting supernatants were then diluted in water containing $0.1 \%$ propionic acid and $0.5 \%$ dimethyl sulfoxide. M4 formation was quantified by LC-MS/MS analysis using an authentic M4 standard.

\subsection{Characterization of Renal Clearance in Animal Models}

Male CD-1 mice $(n=15)$, male Wistar-Hannover rats $(n=6)$, female Dutch Belted rabbits $(n=3)$, and rhesus monkeys $(n=3)$ were administered $1 \mathrm{mg} / \mathrm{kg}$ islatravir intravenously. Blood samples were collected at specified time intervals following dose administration as were urine samples throughout the study period for each animal model; 0-24 $\mathrm{h}$ for mice, rats, and monkeys and $0-48 \mathrm{~h}$ for rabbits. Islatravir concentrations in plasma and urine were determined by LC-MS/MS, following a protein precipitation step. Renal clearance was calculated by dividing the amount of unchanged islatravir excreted into urine over the course of the study by the corresponding area under the plasma-concentration time curve $\left(\mathrm{AUC}_{0-\mathrm{x}}\right)$ in plasma. $\mathrm{AUC}_{0-\mathrm{x}}$ was determined using the linear trapezoidal method for ascending concentrations, and the log trapezoidal method for descending concentrations, and the amount of unchanged islatravir excreted into urine was obtained by multiplying 
the concentration of islatravir in urine by the volume of urine collected over the specified time interval.

\subsection{Interaction of Islatravir with Drug-Metabolizing Enzymes: CYP Isoforms and UGT1A1}

Reversible CYP inhibition was performed in pooled human liver microsomes incubated at $37^{\circ} \mathrm{C}$ in a reaction mixture containing the appropriate CYP probe substrate and islatravir ( 0.05 to $100 \mu \mathrm{M}$ except CYP3A4, which was tested to $200 \mu \mathrm{M})$, as previously reported [55]. Similarly, the potential for islatravir $(0.78-100 \mu \mathrm{M})$ to inhibit the UGT1A1-mediated glucuronidation of estradiol was measured in pooled human liver microsomes, as previously described [55]. CYP2C19 S-mephenytoin $(30 \mu \mathrm{M}) 4^{\prime}$-hydroxylation and CYP2D6 dextromethorphan $(10 \mu \mathrm{M})$ O-demethylation were assessed over incubation periods of $20 \mathrm{~min}$ and used the control inhibitors benzyl-nirvanol and quinidine, respectively. CYP1A2 phenacetin $(100 \mu \mathrm{M})$ O-deethylation, CYP2B6 bupropion $(180 \mu \mathrm{M})$ hydroxylation, CYP2C9 diclofenac $(10 \mu \mathrm{M}) 4^{\prime}$-hydroxylation, and CYP3A4 testosterone $(50 \mu \mathrm{M}) 6 \beta$-hydroxylation were assessed over incubation periods of $10 \mathrm{~min}$, and used the control inhibitors $\alpha$-naphtholflavone, ticlopidine, sulfaphenazole, and ketoconazole, respectively. CYP2C8 amodiaquine $(4 \mu \mathrm{M}) \mathrm{N}$-deethylation and CYP3A4 midazolam $(3 \mu \mathrm{M})$ $1^{\prime}$-hydroxylation were assessed over incubation periods of $3 \mathrm{~min}$, and used the control inhibitors montelukast and ketoconazole, respectively.

The time-dependent inhibition of major human CYP isoforms (1A2, 2B6, 2C8, 2C9, $2 \mathrm{C} 19,2 \mathrm{D} 6$, or 3A4) was performed in pooled human liver microsomes at islatravir concentrations of 10 and $50 \mu \mathrm{M}$, using selective probe substrates for each CYP as previously described [55]. CYP-specific probe substrates were phenacetin (300 $\mu \mathrm{M}$; incubation time $20 \mathrm{~min}$ ) for CYP1A2, efavirenz ( $30 \mu \mathrm{M}$; incubation time $25 \mathrm{~min}$ ) for CYP2B6, amodiaquine ( $20 \mu \mathrm{M}$; incubation time $4 \mathrm{~min})$ for CYP2C8, diclofenac ( $50 \mu \mathrm{M}$; incubation time $12 \mathrm{~min}$ ) for CYP2C9, S-mephenytoin ( $225 \mu \mathrm{M}$; incubation time $25 \mathrm{~min})$ for CYP2C19, bufuralol (50 $\mu \mathrm{M}$; incubation time $15 \mathrm{~min})$ for CYP2D6, and testosterone ( $250 \mu \mathrm{M}$; incubation time $10 \mathrm{~min})$ for CYP3A4. Positive control incubations using a CYP isoform-specific time-dependent inhibitor, control incubations without inhibitor (containing $1 \% v / v$ methanol only), and incubations without NADPH in the inactivation reactions were also performed to assess the overall time-dependent inhibition potential of islatravir.

The potential for islatravir to induce CYP1A2, CYP2B6, and CYP3A4 was assessed in cryopreserved hepatocytes from three human donors, after $48 \mathrm{~h}$ incubation with islatravir (0.1-20 $\mu \mathrm{M})$. The method was as previously described [55], with the exception that all solutions were replaced after $24 \mathrm{~h}$ of incubation. Positive control inducers rifampicin $(10 \mu \mathrm{M})$, phenobarbital $(1000 \mu \mathrm{M})$ or omeprazole $(50 \mu \mathrm{M})$ were used, and after the $48-\mathrm{h}$ incubation period, whole cell-based CYP3A4, CYP2B6, and CYP1A2 enzyme changes were evaluated using testosterone $6 \beta$-hydroxylation, bupropion hydroxylation, and phenacetin O-deethylation, respectively, measured by LC-MS/MS detection. Total RNA was isolated for quantitative polymerase chain reaction analysis of CYP3A4, CYP2B6, and CYP1A2 mRNA expression, as described previously [56]. The overall induction potential of islatravir was then assessed based on regulatory agency recommendations [14-16], which state the induction potential of an investigational drug should not be ruled out if increases in CYP enzyme mRNA were more than $20 \%$ of the response of the positive control.

\subsection{Evaluation of Islatravir as a Perpetrator of Drug-Drug Interactions via Transporters}

Inhibition of recombinant transporter-mediated uptake of probe substrates was determined in recombinant cell lines; HEK293-OATP1B1, HEK293-OATP1B3, CHO-K1-OCT1, CHO-K1-OCT2, MDCKII-OAT1, MDCKII-OAT3, CHO-K1-MATE1, and MDCKII-MATE2K, as described previously $[55,57,58]$, with some modifications to OATP1B1 and OATP1B3 inhibition assays. For these assays, cryopreserved HEK293-OATP1B1 and HEK293-OATP1B3 cell aliquots, used under license agreement from SOLVO ${ }^{\circledR}$ Biotechnology (Hungary), were thawed, recovered, and re-suspended in 96-well glass-coated plates at a density of $0.125 \times 10^{6}$ cells/well with various concentrations of islatravir or cyclosporin $\mathrm{A}$, a 
positive-control inhibitor, under $5 \% \mathrm{CO}_{2}$ at $37{ }^{\circ} \mathrm{C}$ for $30 \mathrm{~min}$. Uptake was then initiated by addition of probe substrates as previously described [58], with the exception that $0.1 \mu \mathrm{M}\left[{ }^{3} \mathrm{H}\right]$ pitavastatin was used as probe substrate for OATP1B1 and uptake time of OATP1B1 and OATP1B3 inhibition assays was $2 \mathrm{~min}$. Pyrimethamine $(5 \mu \mathrm{M})$ was used as a positive control inhibitor in MATE1 and MATE2K inhibition studies. Transportermediated uptake was calculated by subtracting the uptake rate in control cells from that in transporter-expressing cells. Data were normalized to \% control, where uptake in the absence of test compound was $100 \%$.

The inhibitory effect of islatravir $(0.22-200 \mu \mathrm{M})$ on MDR1 P-gp-mediated bi-directional transport of $\left[{ }^{3} \mathrm{H}\right]$-digoxin $(0.1 \mu \mathrm{M})$ was assessed in LLC-PK1 cell lines stably expressing MDR1 P-gp, as previously described [55].

Inhibition of BCRP-mediated $\left[{ }^{3} \mathrm{H}\right]$ methotrexate uptake was assessed in BCRP-containing Sf9 membrane vesicles as previously described [58]. Briefly, $\left[{ }^{3} \mathrm{H}\right]$ methotrexate was mixed with BCRP vesicles (Thermo Fisher Scientific, Waltham, MA, USA) and various concentrations of islatravir or $10 \mu \mathrm{M} \mathrm{Ko} 143$, and preincubated at $37^{\circ} \mathrm{C}$ for $5 \mathrm{~min}$. Uptake was initiated by adding adenosine triphosphate (ATP) or adenosine monophosphate (AMP), followed by incubation at $37^{\circ} \mathrm{C}$ for $5 \mathrm{~min}$. Uptake was stopped and samples transferred to pre-wetted 96-well glass-fiber filter plates, and vacuum was applied. The washing steps and sample analysis were performed as previously described [58]. Similarly, inhibition of BSEP, MRP2-, MRP3-, and MRP4-mediated uptake of probe substrates $\left[{ }^{3} \mathrm{H}\right]$ taurocholic acid $(1 \mu \mathrm{M}),\left[{ }^{14} \mathrm{C}\right]$ ethacrynic acid glutathione conjugate $(1 \mu \mathrm{M}),\left[{ }^{3} \mathrm{H}\right]$ estradiol $17 \beta$-D-glucuronide $(1 \mu \mathrm{M})$, and $\left[{ }^{3} \mathrm{H}\right]$ folic acid $(10 \mu \mathrm{M})$, respectively, was evaluated in Sf9 membrane vesicles containing BSEP, MRP2, MRP3, or MRP4 (Thermo Fisher Scientific, Waltham, MA, USA), in the presence or absence of various concentrations of islatravir, or $100 \mu \mathrm{M}$ atorvastatin (BSEP) or $100 \mu \mathrm{M}$ bromosulfophthalein (MRP2, MRP3, or MRP4) as control inhibitors. Transporter-mediated uptake was calculated by subtracting the uptake in the presence of AMP from that in the presence of ATP, and data were normalized to percent control, where uptake in the absence of test compound was $100 \%$.

\subsection{Evaluation of Islatravir as a Victim of Drug-Drug Interactions via Transporters}

To assess uptake in recombinant transporter expressing cell lines, uptake of $1 \mu \mathrm{M}$ $\left[{ }^{3} \mathrm{H}\right]$ islatravir into MDCKII, MDCKII-OAT1, MDCKII-OAT3, CHO-K1, and CHO-K1-OCT2 cells and $2 \mu \mathrm{M}\left[{ }^{14} \mathrm{C}\right]$ islatravir into CHO-K1, CHO-K1-MATE1, MDCKII, and MDCKIIMATE2K cells was measured using the methods reported previously, with a cell density of $0.4 \times 10^{6}$ cells/well [57]. $\left[{ }^{3} \mathrm{H}\right] p$-aminohippuric acid $(1 \mu \mathrm{M}),\left[{ }^{3} \mathrm{H}\right]$ estrone sulfate $(1 \mu \mathrm{M})$, and $\left[{ }^{14} \mathrm{C}\right]$ tetraethylammonium $(1 \mu \mathrm{M})$ were used as positive control substrates of OAT1, OAT3, and OCT2, respectively. $\left[{ }^{14} \mathrm{C}\right]$ Metformin $(5 \mu \mathrm{M})$ was used as control substrate of MATE1 and MATE2K. Probenecid (1 mM) was used as control inhibitor of OAT1 and OAT3. Quinidine $(0.1 \mathrm{mM})$ was used as control inhibitor of OCT2, and pyrimethamine $(5 \mu \mathrm{M})$ as control inhibitor of MATE1 and MATE2K. Based on internal assay calibrations, and in line with regulatory agency recommendations $[15,16]$, islatravir was considered a transporter substrate when uptake was time-dependent, inhibited by the control transporter inhibitor, and 1.5-fold higher in the transporter-expressing cell line compared with the control cell line, at a minimum of 2 time-points.

To study uptake in MDR1 P-gp-containing membrane vesicles, the time- and ATPdependent uptake of $\left[{ }^{14} \mathrm{C}\right]$ islatravir was measured in control and MDR1 P-gp-containing Sf9 membrane vesicles (Thermo Fisher Scientific, Waltham, MA, USA) [57]. Briefly, $\left[{ }^{14} \mathrm{C}\right]$ islatravir $(5 \mu \mathrm{M})$ or $\left[{ }^{3} \mathrm{H}\right] \mathrm{N}$-methylquinidine $(0.5 \mu \mathrm{M})$, with or without cyclosporin $\mathrm{A}(10 \mu \mathrm{M})$ was pre-incubated with ATP-regenerating reagent or AMP reagent for 5 min at $37^{\circ} \mathrm{C}$. Uptake was initiated by the addition of substrate solution to MDR1 P-gp, or control vesicles, followed by incubation at $37^{\circ} \mathrm{C}$ for $0-10 \mathrm{~min}$. Uptake was stopped and samples transferred to a pre-wetted 96 -well glass fiber filter plate, and vacuum was applied. The washing steps and sample analysis were performed as previously described $[55,57]$. Based on internal assay calibrations, and in line with regulatory agency recommendations $[15,16]$, 
islatravir was considered a substrate of MDR1 P-gp when uptake was time-dependent, inhibited by the control transporter inhibitor cyclosporin A, and 1.5-fold higher in the presence of ATP compared with its absence, at a minimum of 2 time-points.

Bidirectional transport of islatravir $(2 \mu \mathrm{M})$, with or without Ko143 $(5 \mu \mathrm{M}$, a prototypic BCRP inhibitor), was measured across MDCKII and MDCKII-BCRP cell monolayers as previously described [55,57]. Prazosin $(1 \mu \mathrm{M})$, with or without $5 \mu \mathrm{M}$ Ko143, was used as the positive control. Samples were analyzed quantitatively by LC-MS/MS. The apparent permeability $\left(\mathrm{P}_{\text {app }}\right)$ and efflux ratios were calculated as described below and as previously reported $[57,59]$. Based on internal assay calibrations, and in line with regulatory agency recommendations $[15,16]$, islatravir was considered a substrate of BCRP when the folddifference in efflux ratio between the control and BCRP expressing cells was $>2$ and inhibited by the control transporter inhibitor Ko143.

$$
\begin{gathered}
\mathrm{P}_{\mathrm{app}}=\frac{\text { Volume of Receptor Chamber }(\mathrm{mL})}{\left[\text { Area of Membrane }\left(\mathrm{cm}^{2}\right)\right][\text { Initial Concentration }(\mu \mathrm{M})]} \times \frac{\Delta \text { in Concentration }(\mu \mathrm{M})}{\Delta \text { in Time }(\mathrm{s})} \\
\text { Efflux ratio }=\frac{\mathrm{P}_{\mathrm{app}}(\mathrm{B} \text { to } \mathrm{A})}{\mathrm{P}_{\mathrm{app}}(\mathrm{A} \text { to B })}
\end{gathered}
$$

\section{Results}

\subsection{Islatravir Exhibited Low Plasma Protein Binding}

The in vitro assessment indicated that the reversible binding of islatravir to proteins in human plasma was low, with mean unbound fractions in plasma of $0.96 \pm 0.04$ ( \pm standard deviation) at $0.1 \mu \mathrm{M}$ islatravir. Islatravir, at $0.1 \mu \mathrm{M}$, also exhibited low binding to plasma proteins from mouse, rat, rabbit, and monkey, with unbound fractions exceeding 0.84 across species. Islatravir binding to plasma proteins did not exhibit concentration dependence at concentrations ranging between 0.1 and $10 \mu \mathrm{M}$, for all species tested.

The partitioning of islatravir between human red blood cells and plasma was evaluated in vitro, with a mean blood:plasma concentration ratio of $1.3 \pm 0.0$ ( \pm standard error) at $0.1 \mu \mathrm{M}$; islatravir did not exhibit concentration-dependent partitioning over the 0.01 to $10 \mu \mathrm{M}$ concentration range.

\subsection{Islatravir Was Metabolized by Adenosine Deaminase}

In cryopreserved human hepatocyte incubations, no detectable metabolites of islatravir were observed; however, additional in vitro studies have shown that islatravir is extensively metabolized by ADA. Considering the high expression of ADA in the human intestine (compared with the liver) [60], this tissue was used to assess islatravir metabolism. In human intestinal S9 fraction, $\left[{ }^{3} \mathrm{H}\right]$ islatravir exhibited low turnover, with $4^{\prime}$-ethynyl-2fluoro-2'-deoxyinosine (M4; Figure 1B) being the only metabolite detected by LC-MS/MS, interfaced with online radiometric detection. The ADA-mediated metabolism of islatravir was further studied in vitro by incubation with recombinant human ADA. In these studies, very slow ADA-dependent deamination to M4 was observed, which is consistent with previously reported results for islatravir [61]. Enzyme kinetics for recombinant human ADA showed that the rate of M4 formation increased linearly over the 1-250 $\mu \mathrm{M}$ islatravir concentration range, indicating that recombinant ADA has a relatively low affinity for islatravir (Michaelis constant $\left[\mathrm{K}_{\mathrm{m}}\right]>250 \mu \mathrm{M}$ ), consistent with ADA being a high-capacity enzyme.

\subsection{Islatravir Was Partially Cleared via Renal Excretion in Nonclinical Species}

In addition to ADA-mediated metabolism, nonclinical studies demonstrated renal clearance of islatravir to be $15.4,11.5,5.3$, and $8.7 \mathrm{~mL} / \mathrm{min} / \mathrm{kg}$ in mouse, rat, rabbit, and monkey, respectively. The renal excretion of unchanged islatravir contributed $61 \%, 17 \%$, $31 \%$, and $51 \%$ of the total plasma clearance in mouse, rat, rabbit, and monkey models, respectively (Table 1). For all species tested, renal clearance exceeded the glomerular 
filtration rate, suggesting that in addition to filtration, renal clearance of islatravir is partially mediated by active transport in these species. Based on these findings, renal excretion of unchanged islatravir is anticipated to contribute to the overall elimination in humans.

Table 1. Renal clearance and excretion in nonclinical species.

\begin{tabular}{ccc}
\hline Species & $\begin{array}{c}\text { Renal Clearance } \\
(\mathbf{m L} / \mathbf{m i n} / \mathbf{k g})\end{array}$ & $\begin{array}{c}\text { Renal Excretion } \\
\text { (\% Total Plasma Clearance) }\end{array}$ \\
\hline Mouse & 15.4 & 61 \\
Rat & 11.5 & 17 \\
Rabbit & 5.3 & 31 \\
Rhesus macaque & 8.7 & 51 \\
\hline
\end{tabular}

\subsection{Islatravir Did Not Inhibit or Induce Major Drug-Metabolizing Enzymes}

The inhibitory effect of islatravir on CYP isoforms and UGT1A1 in pooled human liver microsomes is summarized in Table 2. No reversible inhibition was observed with islatravir on any CYP isoform tested (1A2, 2B6, 2C8, 2C9, 2C19, 2D6, or 3A4) at concentrations up to $100 \mu \mathrm{M}$, indicating a half maximal inhibitory concentration $\left(\mathrm{IC}_{50}\right)$ greater than $100 \mu \mathrm{M}$ for all reactions. Islatravir concentrations of up to $200 \mu \mathrm{M}$ did not inhibit CYP3A4, indicating an $\mathrm{IC}_{50}$ greater than $200 \mu \mathrm{M}$. Pre-incubation of 10 and $50 \mu \mathrm{M}$ islatravir for up to $30 \mathrm{~min}$ in human liver microsomes caused no time-dependent inhibition of CYP1A2, 2B6, 2C8, 2C9, 2C19, 2D6, and 3A4. No inhibition of UGT1A1-mediated estradiol 3-glucuronidation was observed with islatravir up to $100 \mu \mathrm{M}$ in human liver microsomes, indicating an $\mathrm{IC}_{50}$ greater than $100 \mu \mathrm{M}$.

The potential for islatravir to induce CYP3A4, CYP2B6, or CYP1A2 was assessed in cryopreserved hepatocytes from three donors, after $48 \mathrm{~h}$ exposure to $0.1-20 \mu \mathrm{M}$ islatravir. The mRNA response for CYP3A4, CYP2B6, or CYP1A2 was less than that of the vehicle control for each tested concentration of islatravir (Table 3), which was less than $20 \%$ of respective positive controls, indicating islatravir was not an inducer of these enzymes [14-16]. In line with these data, corresponding enzyme activity of CYP3A4, CYP2B6, or CYP1A2 in the same incubations was not greater than 1.1-fold of solvent control for all islatravir concentrations and CYP isoforms tested.

Table 2. Drug-drug interaction risk calculation for islatravir.

\begin{tabular}{|c|c|c|c|c|c|}
\hline \multicolumn{6}{|c|}{ Islatravir Risk of Interaction with Metabolic Enzymes } \\
\hline Enzyme & $\begin{array}{l}\text { Mechanism of } \\
\text { Inhibition }\end{array}$ & $\begin{array}{l}\text { Islatravir } \mathrm{IC}_{50} \\
(\mu \mathrm{M})^{\mathrm{a}}\end{array}$ & $\begin{array}{c}\text { Maximum } \\
\text { Unbound Plasma } \\
\text { Concentration }{ }^{b} \\
\left(I_{\text {max, }}\right) \text { to } K_{i, u}{ }^{c} \\
\text { Ratio }(\mu \mathrm{M})\end{array}$ & $\begin{array}{l}\text { Intestinal } \\
\text { Concentration } \\
\left(I_{\text {gut }}\right) \text { to } K_{i, u} c \\
\text { Ratio }(\mu M)\end{array}$ & DDI Potential ${ }^{f}$ \\
\hline $\begin{array}{l}\text { CYP1A2, 2B6, 2C8, } \\
\text { 2C9, 2C19, 2D6 }\end{array}$ & Reversible & $>100$ & $<0.019$ & $\mathrm{~N} / \mathrm{A}$ & Low risk \\
\hline CYP3A4 & Reversible & $>200$ & $<0.010$ & $<8.2$ & Low risk \\
\hline UGT1A1 & Reversible & $>100$ & $\mathrm{~N} / \mathrm{A}$ & $<16.4$ & Low risk $\mathrm{g}$ \\
\hline $\begin{array}{c}\text { CYP1A2, 2B6, 2C8, } \\
\text { 2C9, 2C19, 2D6, } \\
3 \mathrm{~A} 4\end{array}$ & Time dependent & $>50$ & $\mathrm{~N} / \mathrm{A}$ & $\mathrm{N} / \mathrm{A}$ & Low risk ${ }^{h}$ \\
\hline
\end{tabular}


Table 2. Cont.

\begin{tabular}{|c|c|c|c|c|c|}
\hline \multicolumn{6}{|c|}{ Islatravir Risk of Interaction with Drug Transporters } \\
\hline Transporter & $\begin{array}{c}\text { Islatravir } \mathrm{IC}_{50} \\
(\mu \mathrm{M})^{\mathrm{a}}\end{array}$ & $\begin{array}{c}\text { Maximum } \\
\text { Unbound Plasma } \\
\text { Concentration }{ }^{b} \\
\left(\mathrm{I}_{\text {max, }}\right) \text { to } \mathrm{IC}_{50} \\
\text { Ratio }(\mu \mathrm{M})\end{array}$ & $\begin{array}{l}\text { Intestinal } \\
\text { Concentration } d \\
\left(\mathrm{I}_{\text {gut }}\right) \text { to } \mathrm{IC}_{50} \text { Ratio } \\
(\mu \mathrm{M})\end{array}$ & $\begin{array}{c}\text { Maximum } \\
\text { Unbound Inlet } \\
\text { Concentration } \\
\left(\mathrm{I}_{\mathrm{in}, \mathrm{max}, \mathrm{u}}\right) \text { to } \mathrm{IC}_{50} \\
\text { Ratio }\end{array}$ & DDI Potential $^{f}$ \\
\hline $\begin{array}{c}\text { OATP1B1, } \\
\text { OATP1B3, OCT1 }\end{array}$ & $>300$ & $\mathrm{~N} / \mathrm{A}$ & $\mathrm{N} / \mathrm{A}$ & $<0.035$ & Low risk \\
\hline $\begin{array}{c}\text { OAT1, OAT3, } \\
\text { OCT2 }\end{array}$ & $>100$ & $<0.010$ & $\mathrm{~N} / \mathrm{A}$ & $\mathrm{N} / \mathrm{A}$ & Low risk \\
\hline MATE1, MATE2K & $>75$ & $<0.013$ & $\mathrm{~N} / \mathrm{A}$ & $\mathrm{N} / \mathrm{A}$ & Low risk \\
\hline BCRP & $>100$ & $<0.010$ & $<8.2$ & $\mathrm{~N} / \mathrm{A}$ & Low risk \\
\hline MDR1 P-gp & $>200$ & $<0.005$ & $<4.1$ & $\mathrm{~N} / \mathrm{A}$ & Low risk \\
\hline
\end{tabular}

${ }^{a}$ When either no inhibition or $<50 \%$ inhibition was observed at the highest concentration tested, IC ${ }_{50}$ was assumed to be above the highest concentration tested, for the purpose of risk assessment. Maximum unbound plasma concentration, intestinal concentration, and unbound inlet concentration to $\mathrm{IC}_{50}$ ratios are predicted based on the parameters and calculations below: ${ }^{b} \mathrm{I}_{\max , \mathrm{u}}=\mathrm{C}_{\max } * \mathrm{f}_{\mathrm{u} . \mathrm{p}}$ where $\mathrm{C}_{\max }=1.01 \mu \mathrm{M}$ for $60 \mathrm{mg}$ at steady state and $\mathrm{f}_{\mathrm{u} . \mathrm{p}}=0.96 .{ }^{\mathrm{c}} \mathrm{K}_{\mathrm{i}, \mathrm{u}}$ calculated as $\mathrm{IC}_{50} / 2 * \mathrm{fu}, \mathrm{mic}$, assuming competitive inhibition, assuming worst case scenario with a $K_{i}$ of $50 \mu \mathrm{M}$ and a calculated fu,mic $=1$ [62]. ${ }^{d} I_{\text {gut }}=60 \mathrm{mg}$ islatravir dose $/ 250 \mathrm{~mL}=818 \mu \mathrm{M} .{ }^{e} \mathrm{I}_{\text {in,max,u }}=\mathrm{f}_{\mathrm{u}, \mathrm{b}} *\left(\mathrm{I}_{\mathrm{max}, \mathrm{b}}+\left(\left(\mathrm{F}_{\mathrm{a}} \mathrm{F}_{\mathrm{g}} \times\right.\right.\right.$ $\mathrm{k}_{\mathrm{a}} \times$ Dose) $\left./ \mathrm{Q}_{\mathrm{h}}\right)$ ) where $\mathrm{f}_{\mathrm{u}, \mathrm{b}}=\mathrm{f}_{\mathrm{u}, \mathrm{p}} / \mathrm{R}_{\mathrm{B}}, \mathrm{I}_{\max , \mathrm{b}}=\mathrm{C}_{\max }{ }^{*} \mathrm{R}_{\mathrm{B}}, \mathrm{R}_{\mathrm{B}}=1.3, \mathrm{Q}_{\mathrm{h}}=1617 \mathrm{~mL} / \mathrm{min}$, and assumes $\mathrm{F}_{\mathrm{a}} \mathrm{F}_{\mathrm{g}}=1$ and $\mathrm{k}_{\mathrm{a}}=0.1$ min ${ }^{-1} .{ }^{\mathrm{f}} \mathrm{Risk}$ assessment based on guidance provided by FDA, EMA, and PMDA [14,16,30]. $\mathrm{g}$ Because no inhibition of UGT1A1 was observed at 100 $\mu$ M, the $\mathrm{IC}_{50}$ is considered to be significantly higher than $100 \mu \mathrm{M}$, and thus the $\mathrm{I}_{\text {gut }}$ to $\mathrm{K}_{\mathrm{i}, \mathrm{u}}$ ratio of $<16.4$ is conservative and the potential for interaction at the gut level is considered to be low. ${ }^{\mathrm{h}}$ Because time-dependent inhibition was not observed, determination of $\mathrm{k}_{\text {inact }}$ and $\mathrm{K}_{\mathrm{i}}$ was not warranted, precluding the need for further risk assessment as outlined by agency guidance. N/A: Indicates calculations are not relevant for transporter or enzyme location. BCRP, breast cancer resistance protein; $\mathrm{C}_{\text {max }}$, maximum plasma concentration; $\mathrm{CYP}$, cytochrome $\mathrm{P} 450$; DDI, drug-drug interaction; EMA, European Medicines Agency; FDA, Food and Drug Administration; $F_{a}$, fraction absorbed; F $F_{g}$, intestinal availability; $\mathrm{f}_{\text {u.p }}$, unbound fraction in plasma; $\mathrm{IC}_{50}$, half maximal inhibitory concentration; $\mathrm{I}_{\text {gut }}$, intestinal luminal concentration; $\mathrm{I}_{\text {in,max, }}$, estimated maximum plasma inhibitor concentration at the liver inlet; $\mathrm{I}_{\max , \mathrm{u}}$, maximal unbound plasma concentration; $\mathrm{k}_{\mathrm{a}}$, absorption rate constant; $\mathrm{K}_{\mathrm{i}}$, inhibition constant; MATE, multidrug and toxin extrusion protein; MDR1 P-gp, multidrug resistance protein 1 P-glycoprotein; OAT, organic anion transporter; OATP, organic anion transporting polypeptide; OCT, organic cation transporter; PMDA, Pharmaceuticals and Medical Devices Agency; $\mathrm{Q}_{\mathrm{h}}$, hepatic blood flow rate; $\mathrm{R}_{\mathrm{B}}$, blood-to-plasma ratio; TDI, time-dependent inhibition; UGT1A1, uridine diphosphate glucuronosyltransferase $1 \mathrm{~A} 1$.

Table 3. Effect of islatravir on CYP mRNA in human hepatocytes.

\begin{tabular}{ccccc}
\hline \multirow{2}{*}{ Concentration $[\boldsymbol{\mu M}]$} & & \multicolumn{2}{c}{ mRNA Mean Fold Change \pm SD $^{\mathbf{a}}$} \\
\cline { 2 - 5 } & & CYP3A4 & CYP2B6 & CYP1A2 \\
\hline DMSO (vehicle) & NA & $1.0 \pm 0.0$ & $1.0 \pm 0.0$ & $1.0 \pm 0.0$ \\
\hline Rifampin (control) & 10 & $9.9 \pm 2.7$ & ND & ND \\
\hline Phenobarbitol (control) & 1000 & ND & $18.5 \pm 1.9$ & ND \\
\hline Omeprazole (control) & 50 & ND & ND & $26.4 \pm 8.6$ \\
\hline & 0.1 & $0.6 \pm 0.2$ & $0.5 \pm 0.1$ & $0.4 \pm 0.2$ \\
\cline { 2 - 5 } Islatravir & 0.5 & $0.6 \pm 0.2$ & $0.5 \pm 0.2$ & $0.4 \pm 0.2$ \\
\cline { 2 - 5 } & 1 & $0.6 \pm 0.2$ & $0.7 \pm 0.2$ & $0.5 \pm 0.3$ \\
\cline { 2 - 5 } & 5 & $0.5 \pm 0.1$ & $0.7 \pm 0.1$ & $0.4 \pm 0.3$ \\
\cline { 2 - 5 } & 10 & $0.6 \pm 0.1$ & $0.9 \pm 0.3$ & $0.5 \pm 0.4$ \\
\hline
\end{tabular}

a Mean \pm SD fold change was calculated by dividing mRNA levels in treated samples, by those in the DMSO vehicle control samples, for $n=3$ donors. Fold change for vehicle control was set to 1.0 CYP, cytochrome P450; DMSO, dimethylsulfoxide; NA, not applicable; ND, not determined; SD, standard deviation.

\subsection{Islatravir Did Not Inhibit Major Hepatic Transporters at Clinically Relevant Concentrations}

In recombinant cell lines, concentrations of islatravir of up to $300 \mu \mathrm{M}$ did not inhibit the OATP1B1-, OATP1B3-, and OCT1-mediated uptake of pitavastatin, sulfobromophthalein, or metformin, respectively. Similarly, islatravir concentrations of up to $100 \mu \mathrm{M}$ did not inhibit 
the BSEP-, MRP2-, MRP3-, and MRP4-mediated ATP-dependent uptake of taurocholic acid, ethacrynic acid glutathione conjugate, $E_{2} 17 \beta G$, or folic acid, respectively, in Sf9 membrane vesicles containing these efflux transporters. This indicates $\mathrm{IC}_{50}$ values greater than $300 \mu \mathrm{M}$ for OATP1B1, OATP1B3, and OCT1, and greater than $100 \mu \mathrm{M}$ for the other hepatic transporters tested (Table 2).

\subsection{Islatravir Did Not Inhibit Major Renal Transporters at Clinically Relevant Concentrations}

OAT1-mediated cidofovir uptake in recombinant cell lines was not inhibited by concentrations of islatravir up to $100 \mu \mathrm{M}$, whereas islatravir inhibited OAT3-mediated estrone sulfate uptake and OCT2-mediated metformin uptake by $31 \%$ and $15 \%$ at $100 \mu \mathrm{M}$, respectively. Metformin uptake into recombinant cell lines expressing the renal efflux transporters MATE1 or MATE2K was not inhibited by concentrations of islatravir up to $75 \mu \mathrm{M}$. Taken together, these data indicate that the $\mathrm{IC}_{50}$ values for inhibition of the tested renal drug transporters are greater than $75 \mu \mathrm{M}$ for MATE1 and MATE2K, and greater than $100 \mu \mathrm{M}$ for OAT1, OAT3, and OCT2 (Table 2).

\subsection{Islatravir Did Not Inhibit MDR1 P-gp and BCRP at Clinically Relevant Concentrations}

The potential for islatravir to inhibit the broadly expressed efflux transporters MDR1 P-gp and BCRP was assessed in vitro, in accordance with current regulatory guidance. Islatravir showed no concentration-dependent inhibition of MDR1 P-gp-mediated digoxin transport across monolayers of recombinant cells expressing this transporter, up to $200 \mu \mathrm{M}$, indicating an $\mathrm{IC}_{50}$ greater than $200 \mu \mathrm{M}$. In a membrane vesicle assay, islatravir inhibited $35 \%$ of human BCRP-mediated methotrexate uptake at $100 \mu \mathrm{M}$, indicating an $\mathrm{IC}_{50}$ greater than $100 \mu \mathrm{M}$ (Figure 3).

\section{A}

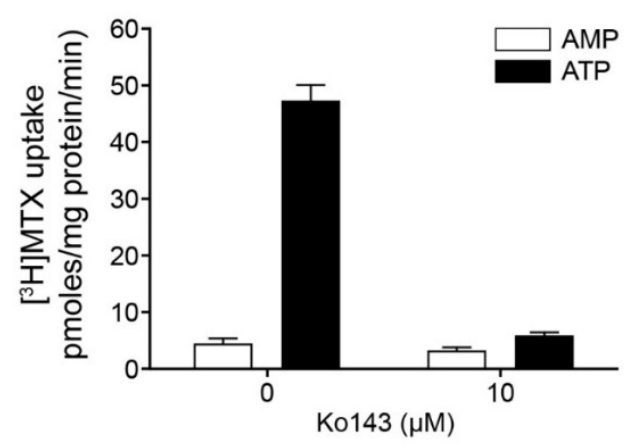

B

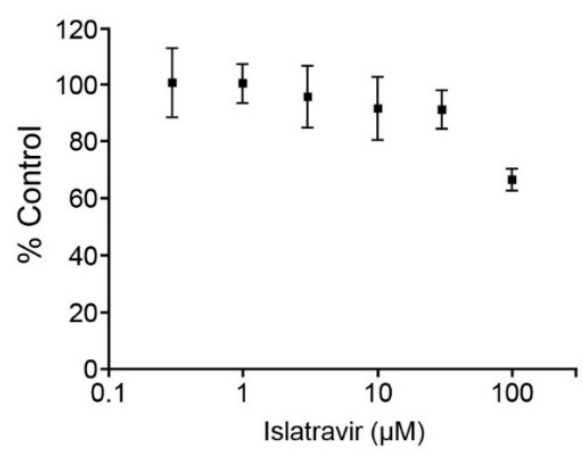

Figure 3. Inhibition of BCRP by islatravir. (A) Positive control, the effect of Ko143 $(10 \mu \mathrm{M})$ on the uptake of $\left[{ }^{3} \mathrm{H}\right]$ methotrexate (MTX $10 \mu \mathrm{M}$ ) in BCRP-containing Sf9 membrane vesicles, in the presence of ATP or AMP. (B) The effect of islatravir on ATP-dependent uptake of $\left[{ }^{3} \mathrm{H}\right]$ methotrexate $(10 \mu \mathrm{M})$ in 
BCRP-containing Sf9 membrane vesicles (percentage of control). The experiment was performed in triplicate. All data are mean $\pm \mathrm{SD}$. AMP, adenosine monophosphate; ATP, adenosine triphosphate; BCRP, breast cancer resistance protein; $\left[{ }^{3} \mathrm{H}\right] \mathrm{MTX},\left[{ }^{3} \mathrm{H}\right]$ methotrexate; SD, standard deviation.

\subsection{Islatravir Was Not a Substrate of Major Renal Transporters}

As islatravir is expected to be partially eliminated by urinary excretion in humans, the transport of islatravir via renal transporters OCT2, OAT1, OAT3, MATE1, and MATE2K was investigated in vitro (Table 4 ). Across the time points tested (1-5 min for OCT2, OAT1, and OAT3, and 5-20 min for MATE1 and MATE2K), uptake of islatravir into recombinant cell lines expressing these transporters at any time point was 0.7 to 1.3 -fold compared with that in parental cells, indicating that islatravir was not a substrate of these renal transporters $[15,16]$. The assays were considered functional as the uptake of the positive control substrates was 6 to 161-fold higher in the transporter-expressing cell lines compared with control cell lines and was fully inhibited by the control transporter inhibitors.

Table 4. Assessment of islatravir as a substrate of renal drug transporters in vitro.

\begin{tabular}{|c|c|c|c|c|}
\hline \multirow{2}{*}{ Transporter } & \multicolumn{2}{|c|}{ Islatravir Uptake ${ }^{a}$ (pmole/10 6 Cells) } & \multirow{2}{*}{ Fold-Difference $^{b}$} & \multirow{2}{*}{ Conclusions } \\
\hline & Control Cells & Transporter-Expressing Cells & & \\
\hline OCT2 & $0.97 \pm 0.01$ & $0.79 \pm 0.14$ & 0.81 & Non-substrate \\
\hline OAT1 & $0.69 \pm 0.07$ & $0.72 \pm 0.04$ & 1.04 & Non-substrate \\
\hline OAT3 & $0.69 \pm 0.07$ & $0.85 \pm 0.06$ & 1.23 & Non-substrate \\
\hline MATE1 & $2.90 \pm 0.27$ & $2.94 \pm 0.20$ & 1.01 & Non-substrate \\
\hline MATE2K & $3.12 \pm 0.17$ & $3.56 \pm 0.17$ & 1.14 & Non-substrate \\
\hline
\end{tabular}

${ }^{a}$ Mean \pm SD, $n=3$ at last time point tested ( 5 min for OCT2, OAT1, OAT3, 10 min for MDR1 P-gp, and 20 min for MATE1 and MATE2K);

${ }^{\mathrm{b}}$ Fold-difference represents ratio of uptake into transporter-expressing cells to control cells; MATE, multidrug and toxin extrusion protein;

OAT, organic anion transporter; OCT, organic cation transporter; SD, standard deviation.

\subsection{Islatravir Was a Substrate of BCRP, but Not MDR1 P-gp}

MDR1 P-gp and BCRP are widely expressed efflux transporters, and thus islatravir was investigated as a substrate of these transporters. Uptake of islatravir into membrane vesicles containing MDR1 P-gp was similar to that in control vesicles (3.7 \pm 1.3 and $5.7 \pm 2.1 \mathrm{pmole} / \mathrm{mg}$ protein (mean \pm standard deviation), respectively in the presence of ATP at the final time point of $10 \mathrm{~min}$ ) and was not ATP-dependent, indicating that islatravir was not a substrate of MDR1 P-gp [15,16]. The assay was considered functional as the uptake of the positive control substrate was 26-fold higher in the presence of ATP, compared with its absence, and was fully inhibited by the control transporter inhibitor.

Islatravir was found to be a substrate of BCRP in a bi-directional transport assay, despite a relatively low apparent permeability coefficient $\left(\mathrm{P}_{\mathrm{app}}\right)$ of $1.6-2.2 \times 10^{6} \mathrm{~cm} / \mathrm{s}$ in MDCKII cells. The mean $\mathrm{P}_{\mathrm{app}}(\mathrm{B}$ to $\mathrm{A}) / \mathrm{P}_{\text {app }}(\mathrm{A}$ to $\mathrm{B})$ efflux ratio for islatravir $(2 \mu \mathrm{M})$ across BCRP-transfected monolayers was $4.3 \pm 1.1$ [mean \pm standard error of the mean $(\mathrm{SEM})]$, compared with a ratio of $0.8 \pm 0.2$ (mean $\pm \mathrm{SEM}$ ) for the untransfected control cells (Figure 4B). Furthermore, this BCRP-mediated transport of islatravir was inhibited by the addition of the prototypic BCRP inhibitor Ko143 $(5 \mu \mathrm{M})$ with the efflux ratio reduced to $0.9 \pm 0.1$ (mean \pm SEM). In comparison, the efflux ratio of the positive control substrate prazosin in BCRP-transfected monolayers was $14.9 \pm 4.9$ (mean $\pm \mathrm{SEM}$ ), compared with a ratio of $1.0 \pm 0.09$ (mean $\pm \mathrm{SEM}$ ) for the untransfected control cells (Figure 4A). 
A

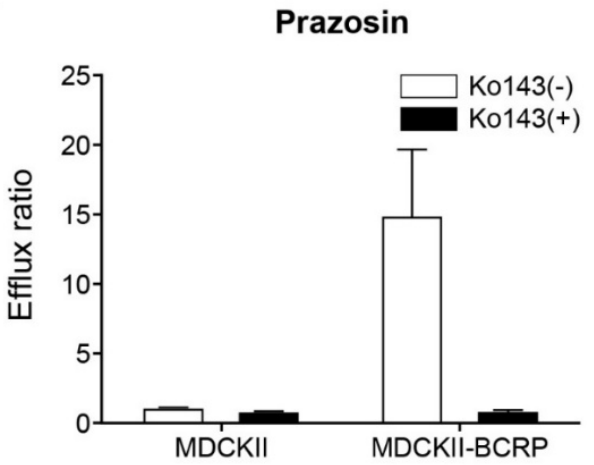

B

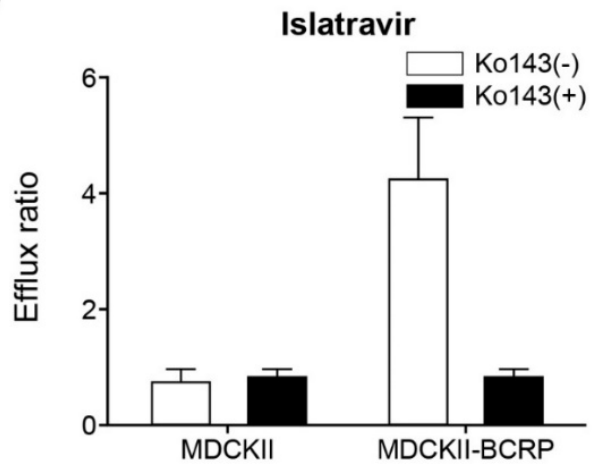

Figure 4. Bidirectional transport of islatravir across MDCKII and MDCKII-BCRP cell monolayers. (A) Positive control, efflux ratio of $1 \mu \mathrm{M}$ prazosin across MDCKII and MDCKII BCRP cell monolayers, in the presence and absence of $5 \mu \mathrm{M} \mathrm{Ko143} \mathrm{(control} \mathrm{inhibitor).} \mathrm{(B)} \mathrm{Efflux} \mathrm{ratio} \mathrm{of} 2 \mu \mathrm{M}$ islatravir across MDCKII and MDCKII-BCRP cell monolayers after 3-h incubation, in the presence and absence of $5 \mu \mathrm{M}$ Ko143. Efflux ratio: $\mathrm{P}_{\text {app }}$ (B to A)/ $\mathrm{P}_{\text {app }}$ (A to B). The experiment was performed in triplicate, except for prazosin in MDCKII without $\operatorname{Ko} 143(n=2)$. All data are mean \pm SEM. BCRP, breast cancer resistance protein; SEM, standard error of the mean.

\section{Discussion}

The in vitro studies reported here characterize the potential drug interaction profile of islatravir, a novel NRTTI in clinical development for the treatment and prevention of HIV-1 $[17,18]$. The drug-metabolizing enzymes and transporters selected for evaluation were based upon the disposition of islatravir and the commonly prescribed medications expected to be taken concomitantly with islatravir (Figure 2), in line with current regulatory guidance and requirements [14-16].

Islatravir was found to have an equal distribution in blood and plasma and low binding to plasma proteins. First-pass metabolism is expected based on the abundance of ADA in the intestine [60]. In vitro assessment of the metabolism of islatravir in intestinal S9 fraction showed inefficient deamination, as observed in the presence of recombinant human ADA. The enzyme kinetics for recombinant human ADA showed a linear rate of M4 formation at concentrations of islatravir between 1 and $250 \mu \mathrm{M}$, which indicated that the ADA-catalyzed metabolism of islatravir to M4 is a high-capacity reaction, with a $\mathrm{K}_{\mathrm{m}}$ greater than $250 \mu \mathrm{M}$. Thus, saturation of ADA-mediated metabolism is not expected at clinically relevant doses of islatravir. Previous studies have shown that the 2-fluoro group in the islatravir structure significantly decreases its susceptibility to hydrolysis by ADA, increasing its intracellular half-life $[18,20,24]$. There was no evidence of islatravir metabolism in human cryopreserved hepatocytes, suggesting that hepatic metabolism may not contribute significantly to the elimination of islatravir. Islatravir was, however, partially eliminated via urinary excretion in animal models and is expected to be the same in humans. 
In the current in vitro analysis, probe drug substrates were used to assess islatravir as a potential perpetrator of metabolizing enzyme and/or transporter-mediated drug-drug interactions. The probe drugs used are known substrates of a given metabolic or transporter pathway $[30,63]$. The pathways by which these probe drugs are metabolized and transported are well established and any observed drug interaction can be applied across other more commonly prescribed agents, which are known to have the same metabolic or transport pathway.

In these studies, the potential interaction of islatravir with major drug-metabolizing enzymes, CYP isoforms, and UGT1A1, was assessed. The results demonstrate no reversible inhibition of CYP3A4 up to $200 \mu \mathrm{M}$ islatravir, indicating an $\mathrm{IC}_{50}$ greater than $200 \mu \mathrm{M}$. For other CYP isoforms and UGT1A1, no reversible inhibition was shown at islatravir concentrations up to $100 \mu \mathrm{M}$, indicating $\mathrm{IC}_{50}$ values greater than $100 \mu \mathrm{M}$. These $\mathrm{IC}_{50}$ values are well above the expected therapeutic $C_{\max }$ of islatravir and exceed the projected $C_{\max }$ of $1.01 \mu \mathrm{M}$ for a $60 \mathrm{mg}$ oral dose by almost two orders of magnitude [36], indicating wide margins to any potential islatravir-mediated effects for doses up to, and including, $60 \mathrm{mg}$ (Table 2).

Hepatic drug-metabolizing enzymes are associated with a large proportion of clinically relevant drug-drug interactions, with CYPs having a role in the metabolism of $70-80 \%$ of drugs [64]. Drugs commonly prescribed in PLWH metabolized by CYPs and UGT1A1 include the proton-pump inhibitor omeprazole, the antiplatelet drug clopidogrel, the selective serotonin reuptake inhibitor citalopram, the opioid buprenorphine, and the antibiotic rifampin, amongst others [30,37-39,43-45,47-49,51-53,65,66].

No time-dependent inhibition by islatravir was observed for CYP1A2, 2B6, 2C8, 2C9, 2C19, 2D6, and 3A4. CYP3A4 is the most abundantly expressed drug-metabolizing enzyme in humans most commonly associated with drug interactions. CYP3A4 is responsible for the metabolism of numerous drugs, including the benzodiazepine alprazolam, atorvastatin, antihistamines, and a majority of antiretroviral agents $[30,63,66]$.

In addition to drug-metabolizing enzymes, drug transporters play an important role in drug distribution and elimination; thus, the impact of islatravir on major uptake and efflux transporters, and the effect of these transporters on islatravir, was assessed. Islatravir demonstrated no inhibitory effect on hepatic uptake transporters OATP1B1, OATP1B3, and OCT1, which are essential for the uptake of major drugs, such as statins and angiotensin II receptor blockers, from sinusoidal blood into the liver for clearance [67]. At the $60 \mathrm{mg}$ dose, the projected maximum free concentration of islatravir at the liver inlet is approximately $10 \mu \mathrm{M}$, which is more than 30-fold lower than the maximum concentration of islatravir for which there was no inhibition of hepatic uptake transporters in these studies (Table 2). Cardiovascular disease and diabetes are increasing in prevalence in PLWH $[2,7,8,30]$; importantly, the commonly prescribed drugs to treat these conditions, including atorvastatin, rosuvastatin, angiotensin II receptor blockers, and metformin, which are hepatic uptake transporter substrates, are not anticipated to interact with islatravir. Islatravir also demonstrated no inhibitory effect on the hepatic efflux transporters BSEP, MRP2, MRP3, and MRP4, which are involved in the hepatic efflux of endogenous bile acids $[67,68]$. Inhibition of these transporters, particularly BSEP, is associated with druginduced liver injury and cholestasis [33,69].

Considering the anticipated contribution of renal excretion in the elimination of islatravir in humans, the lack of metabolism of islatravir observed in human hepatocytes, and the low expression of ADA in the liver [60], hepatic metabolism is not expected to be a significant route of elimination; therefore, islatravir was not assessed as a substrate of hepatic drug-metabolizing enzymes or uptake transporters.

Renal uptake transporters, including OAT1, OAT3, and OCT2, are involved in the elimination of commonly prescribed medications, such as metformin, antiarrhythmics, and diuretics, as well as multiple antibiotics and antiviral drugs, such as adefovir, ganciclovir, and tenofovir $[30,70]$. Tenofovir disoproxil fumarate is a nucleoside reverse transcriptase inhibitor that is metabolized by plasma and tissue esterases to tenofovir [71], which is 
actively transported by OAT1 and OAT3 into renal proximal tubule cells and then eliminated into the urine by MRP2 and MRP4. Inhibition of these transporters may lead to drug accumulation and renal toxicity [72]. At clinically relevant concentrations, islatravir did not inhibit OAT1, OAT3, or OCT2, with $\mathrm{IC}_{50}$ values greater than $100 \mu \mathrm{M}$. Furthermore, islatravir was not found to be a substrate of these transporters. In addition, islatravir was neither a substrate nor an inhibitor of the renal efflux transporters MATE1, MATE2K, and MDR1 P-gp. This finding indicates that islatravir is not likely to be either the perpetrator or victim of renal transporter-based drug-drug interactions with renal uptake substrates or inhibitors, such as the HIV integrase strand transfer inhibitor dolutegravir and the histamine-2 receptor antagonist cimetidine [30,70]. The $\mathrm{IC}_{50}$ values for the interactions between islatravir and major renal transporters exceed the projected maximum unbound plasma concentrations for a $60 \mathrm{mg}$ dose by approximately 100-fold [73], indicating wide margins for dosing without the consideration for drug-drug interactions (Table 2).

Islatravir was not found to be an inhibitor of BCRP at clinically meaningful concentrations (Table 2); however, it was found to be a substrate of BCRP in vitro (Figure 3). Unlike other substrates of BCRP such as rosuvastatin and sulfasalazine [32], islatravir is unlikely to be the victim of BCRP-mediated drug-drug interactions due to its good absorption in vivo, and an anticipated lack of major hepatic secretory clearance [26,74]. Should BCRP contribute to the intestinal efflux of islatravir in vivo, co-administration of an inhibitor of BCRP would only serve to increase absorption of islatravir, which is already well absorbed and is expected to have a favorable drug-drug interaction and toxicity profile [26,74].

Together, these findings are in good agreement with clinical studies conducted to date that demonstrated a lack of drug-drug interactions between islatravir and other agents in participants without HIV. A PK and safety study of islatravir co-administered with doravirine, which is primarily metabolized by CYP3A4, demonstrated no clinically meaningful effects on the PK of either drug [54,75]. Another PK and safety study demonstrated no meaningful drug-drug interactions between islatravir and tenofovir disoproxil fumarate, which is eliminated renally via OAT1 and OAT3, and dolutegravir, which is hepatically metabolized by UGT enzymes and CYP3A4 [70,71,76]. No significant drug-drug interactions have been observed following co-administration of islatravir with levonorgestrel/ethinyl estradiol [77], common components of hormonal contraceptives that are extensively metabolized by CYP3A4, are glucuronidated, and undergo biliary and urinary excretion [78].

Due to its high potency and long intracellular half-life, islatravir remains efficacious at very low doses. Combined with its lack of inhibition of major metabolizing enzymes and drug transporters, islatravir has low potential for drug-drug interactions. Using static drug-drug interaction risk assessment models based on regulatory agency guidelines, islatravir is considered at low risk of drug-drug interactions with major drug transporters and drug-metabolizing enzymes due to the low exposures at therapeutic doses and the lack of inhibition observed in vitro [14,15,79] (Table 2).

\section{Conclusions}

The lack of interaction of islatravir with major drug-metabolizing enzymes and drug transporters and their substrates reinforces the favorable drug-drug interaction profile of islatravir and its potential to be administered as part of combination ART and alongside concomitant medications. This finding is of particular clinical relevance for PLWH who may require polypharmacy for the management of both HIV and common comorbidities, such as diabetes, cardiovascular disease, and depression. Islatravir is not expected to interact with the major pathways associated with other antiretroviral agents, including dolutegravir, doravirine, and tenofovir disoproxil fumarate $[54,71,76]$ as well as with commonly prescribed medications, including metformin, omeprazole, clopidogrel, statins, alprazolam, buprenorphine/naloxone, selective serotonin reuptake inhibitors, oral contraceptives, and rifampin [77]. These results support the continued clinical evaluation of islatravir as an option across diverse populations for the treatment and prevention of HIV-1 infection. 
Author Contributions: K.B., R.H., B.L., M.H., J.G., M.L. and K.L.F. substantially contributed to the conception, design or planning of the study. R.H., J.G., M.H., B.L. and M.L. substantially contributed to the acquisition of the data. K.B., K.L.F., R.H., J.G., M.H., B.L. and M.L. substantially contributed to analysis of the data. K.B., K.L.F., R.H., J.G., M.H., B.L., M.L. and R.I.S. substantially contributed to interpretation of the results. All authors critically reviewed or revised the manuscript for important intellectual content. All authors have read and agreed to the published version of the manuscript.

Funding: Funding for this study was provided by Merck Sharp \& Dohme Corp., a subsidiary of Merck \& Co., Inc., Kenilworth, NJ, USA.

Institutional Review Board Statement: All animal studies were conducted following guidelines and procedures approved by the Institutional Animal Care and Use Committee (IACUC), Merck Sharp \& Dohme Corp., a subsidiary of Merck \& Co., Inc., Kenilworth, NJ, USA.

Informed Consent Statement: Not applicable.

Data Availability Statement: Available data can be obtained by contacting the corresponding author. Any data that are reasonably requested will be made available in a timely fashion to members of the scientific community with as few restrictions as feasible for noncommercial purposes. Subject to requirements or limitations imposed by local and/or U.S. government laws and regulations, we will make every effort to provide to those that request it additional information on the sources of materials used in the studies described here.

Acknowledgments: The authors would like to thank Merck employees Deborah Newton for oversight and review of CYP inhibition and induction data, and Joan Ellis for bioanalytical support for the renal excretion studies. Medical writing and editorial assistance, under the direction of the authors, were provided by Matthew Broadbent and Jared C. Cochran in accordance with Good Publication Practice (GPP3) guidelines. This assistance was funded by Merck Sharp \& Dohme Corp., a subsidiary of Merck \& Co., Inc., Kenilworth, NJ, USA (MSD).

Conflicts of Interest: K.B., R.H., M.H., M.L., J.G., B.L., R.I.S. and K.L.F. are current or former employees of and may own stock and/or stock options in Merck Sharp \& Dohme Corp., a subsidiary of Merck \& Co., Inc., Kenilworth, NJ, USA (MSD).

\section{References}

1. Antiretroviral Therapy Cohort Collaboration. Life expectancy of individuals on combination antiretroviral therapy in high-income countries: A collaborative analysis of 14 cohort studies. Lancet 2008, 372, 293-299. [CrossRef]

2. Marcus, J.L.; Leyden, W.A.; Alexeeff, S.E.; Anderson, A.N.; Hechter, R.C.; Hu, H.; Lam, J.O.; Towner, W.J.; Yuan, Q.; Horberg, M.A.; et al. Comparison of Overall and Comorbidity-Free Life Expectancy between Insured Adults with and without HIV Infection, 2000-2016. JAMA Netw. Open 2020, 3, e207954. [CrossRef]

3. UNAIDS. UNAIDS Fact Sheet-Global AIDS Update 2019. Available online: https://www.unaids.org/sites/default/files/ media_asset/UNAIDS_FactSheet_en.pdf (accessed on 22 July 2021).

4. Wada, N.; Jacobson, L.P.; Cohen, M.; French, A.; Phair, J.; Munoz, A. Cause-specific life expectancies after 35 years of age for human immunodeficiency syndrome-infected and human immunodeficiency syndrome-negative individuals followed simultaneously in long-term cohort studies, 1984-2008. Am. J. Epidemiol. 2013, 177, 116-125. [CrossRef] [PubMed]

5. Lerner, A.M.; Eisinger, R.W.; Fauci, A.S. Comorbidities in Persons with HIV: The Lingering Challenge. JAMA 2019, 323, 19-20. [CrossRef] [PubMed]

6. Smit, M.; Brinkman, K.; Geerlings, S.; Smit, C.; Thyagarajan, K.; Sighem, A.; de Wolf, F.; Hallett, T.B.; Cohort, A.O. Future challenges for clinical care of an ageing population infected with HIV: A modelling study. Lancet Infect. Dis. 2015, 15, 810-818. [CrossRef]

7. Friedman, E.E.; Duffus, W.A. Chronic health conditions in Medicare beneficiaries 65 years old, and older with HIV infection. AIDS 2016, 30, 2529-2536. [CrossRef]

8. Guaraldi, G.; Orlando, G.; Zona, S.; Menozzi, M.; Carli, F.; Garlassi, E.; Berti, A.; Rossi, E.; Roverato, A.; Palella, F. Premature age-related comorbidities among HIV-infected persons compared with the general population. Clin. Infect. Dis. 2011, 53, 1120-1126. [CrossRef]

9. Back, D.; Marzolini, C. The challenge of HIV treatment in an era of polypharmacy. J. Int. AIDS Soc. 2020, 23, e25449. [CrossRef]

10. Escota, G.V.; O'Halloran, J.A.; Powderly, W.G.; Presti, R.M. Understanding mechanisms to promote successful aging in persons living with HIV. Int. J. Infect. Dis. 2018, 66, 56-64. [CrossRef]

11. Gallant, J.; Hsue, P.Y.; Shreay, S.; Meyer, N. Comorbidities among US patients with prevalent HIV infection-a trend analysis. J. Infect. Dis. 2017, 216, 1525-1533. [CrossRef] [PubMed]

12. Greene, M.; Steinman, M.A.; McNicholl, I.R.; Valcour, V. Polypharmacy, drug-drug interactions, and potentially inappropriate medications in older adults with human immunodeficiency virus infection. J. Am. Geriatr. Soc. 2014, 62, 447-453. [CrossRef] 
13. Tornio, A.; Filppula, A.M.; Niemi, M.; Backman, J.T. Clinical studies on drug-drug interactions involving metabolism and transport: Methodology, pitfalls, and interpretation. Clin. Pharmacol. Ther. 2019, 105, 1345-1361. [CrossRef] [PubMed]

14. European Medicines Agency. Guideline on the Investigation of Drug Interactions. 2012. Available online: https://www.ema. europa.eu/en/documents/scientific-guideline/guideline-investigation-drug-interactions-revision-1_en.pdf (accessed on 22 July 2021).

15. US Food and Drug Administration Center for Drug Evaluation and Research. Clinical Drug Interaction StudiesCytochrome P450 Enzyme- and Transporter-Mediated Drug Interactions Guidance for Industry. 2020. Available online: https:/ / www.fda.gov/regulatory-information/search-fda-guidance-documents/clinical-drug-interaction-studiescytochrome-p450-enzyme-and-transporter-mediated-drug-interactions (accessed on 22 July 2021).

16. PMDA Ministry of Labor and Welfare Guideline on Drug Interaction for Drug Development and Appropriate Provision of Information, Notification No.0723-4, Pharmaceutical Evaluation Division, Pharmaceuticals Safety and Environmental Health Bureau, Japan. 23 July 2018. Available online: https:/ / www.pmda.go.jp/files/000228122.pdf (accessed on 22 July 2021).

17. Markowitz, M.; Grobler, J.A. Islatravir for the treatment and prevention of infection with the human immunodeficiency virus type 1. Curr. Opin. HIV AIDS 2020, 15, 27-32. [CrossRef]

18. Markowitz, M.; Sarafianos, S.G. 4'-Ethynyl-2-fluoro-2'-deoxyadenosine, MK-8591: A novel HIV-1 reverse transcriptase translocation inhibitor. Curr. Opin. HIV AIDS 2018, 13, 294-299. [CrossRef]

19. Kawamoto, A.; Kodama, E.; Sarafianos, S.G.; Sakagami, Y.; Kohgo, S.; Kitano, K.; Ashida, N.; Iwai, Y.; Hayakawa, H.; Nakata, H.; et al. 2'-deoxy-4'-C-ethynyl-2-halo-adenosines active against drug-resistant human immunodeficiency virus type 1 variants. Int. J. Biochem. Cell Biol. 2008, 40, 2410-2420. [CrossRef] [PubMed]

20. Michailidis, E.; Huber, A.D.; Ryan, E.M.; Ong, Y.T.; Leslie, M.D.; Matzek, K.B.; Singh, K.; Marchand, B.; Hagedorn, A.N.; Kirby, K.A.; et al. 4'-Ethynyl-2-fluoro-2'-deoxyadenosine (EFdA) inhibits HIV-1 reverse transcriptase with multiple mechanisms. J. Biol. Chem. 2014, 289, 24533-24548. [CrossRef] [PubMed]

21. Salie, Z.L.; Kirby, K.A.; Michailidis, E.; Marchand, B.; Singh, K.; Rohan, L.C.; Kodama, E.N.; Mitsuya, H.; Parniak, M.A.; Sarafianos, S.G. Structural basis of HIV inhibition by translocation-defective RT inhibitor $4^{\prime}$-ethynyl-2-fluoro-2'-deoxyadenosine (EFdA). Proc. Natl. Acad. Sci. USA 2016, 113, 9274-9279. [CrossRef] [PubMed]

22. DHHS. Panel on Antiretroviral Guidelines for Adults and Adolescents Guidelines for the Use of Antiretroviral Agents in Adults and Adolescents with HIV. Department of Health and Human Services. 2019. Available online: https://clinicalinfo.hiv.gov / sites/default/files/guidelines/documents / AdultandAdolescentGL.pdf (accessed on 22 July 2021).

23. Grobler, J.; Lai, M.T.; Barrett, S.E.; Gindy, M.; Fillgrove, K.; Ankrom, W.; Sandra, W.; Friedman, E.; Iwamoto, M.; Hazuda, D.J. Long-acting oral and parenteral dosing of MK-8591 for HIV treatment or prophylaxis. In Proceedings of the Conference on Retroviruses and Opportunistic Infections (CROI), Boston, MA, USA, 22-25 February 2016.

24. Michailidis, E.; Marchand, B.; Kodama, E.N.; Singh, K.; Matsuoka, M.; Kirby, K.A.; Ryan, E.M.; Sawani, A.M.; Nagy, E.; Ashida, N.; et al. Mechanism of inhibition of HIV-1 reverse transcriptase by $4^{\prime}$-Ethynyl-2-fluoro-2'-deoxyadenosine triphosphate, a translocation-defective reverse transcriptase inhibitor. J. Biol. Chem. 2009, 284, 35681-35691. [CrossRef] [PubMed]

25. Stoddart, C.A.; Galkina, S.A.; Joshi, P.; Kosikova, G.; Moreno, M.E.; Rivera, J.M.; Sloan, B.; Reeve, A.B.; Sarafianos, S.G.; MurpheyCorb, M.; et al. Oral administration of the nucleoside EFdA (4'-ethynyl-2-fluoro-2'-deoxyadenosine) provides rapid suppression of HIV viremia in humanized mice and favorable pharmacokinetic properties in mice and the rhesus macaque. Antimicrob. Agents Chemother. 2015, 59, 4190-4198. [CrossRef] [PubMed]

26. Schurmann, D.; Rudd, D.J.; Zhang, S.; De Lepeleire, I.; Robberechts, M.; Friedman, E.; Keicher, C.; Huser, A.; Hofmann, J.; Grobler, J.A.; et al. Safety, pharmacokinetics, and antiretroviral activity of islatravir (ISL, MK-8591), a novel nucleoside reverse transcriptase translocation inhibitor, following single-dose administration to treatment-naive adults infected with HIV-1: An open-label, phase 1b, consecutive-panel trial. Lancet HIV 2020, 7, e164-e172. [CrossRef]

27. Matthews, R.P.; Jackson Rudd, D.; Levine, V.; Zhang, S.; Sterling, L.; Grobler, J.A.; Vargo, R.; Stoch, S.A.; Iwamoto, M. Multiple daily doses of MK-8591 as low as $0.25 \mathrm{mg}$ are expected to suppress HIV. In Proceedings of the Conference on Retroviruses and Opportunistic Infections (CROI), Boston, MA, USA, 4-7 March 2018.

28. Clinicaltrials.gov. Safety and Pharmacokinetics of Oral Islatravir (MK-8591) Once Monthly in Participants at Low Risk of Human Immunodeficiency Virus 1 (HIV-1) Infection (MK-8591-016) (NCT04003103). 2020. Available online: https://clinicaltrials.gov/ct2 / show / NCT04003103 (accessed on 22 July 2021).

29. Hillier, S.; Bekker, L.G.; Badal-Faesen, S.; Hendrix, C.W.; Riddler, S.A.; Rasmussen, S.; Schwartz, H.; Nair, G.; Lombaard, J.H.; Caraco, Y.; et al. Trial design, enrollment status, demographics, and pharmacokinetic (PK) data from a blinded interim analysis from a phase 2a trial of islatravir once monthly (QM) for HIV pre-exposure prophylaxis (PrEP). In Proceedings of the Conference on HIV Research for Prevention (HIVR4P), Virtual, 27-28 January 2021 and 3-4 February 2021.

30. US Food and Drug Administration. Drug Development and Drug Interactions: Table of Substrates, Inhbitors and Inducers. 2020. Available online: https:/ / www.fda.gov/drugs/drug-interactions-labeling/drug-development-and-drug-interactions-tablesubstrates-inhibitors-and-inducers\#table2-1 (accessed on 22 July 2021).

31. Hodges, L.M.; Markova, S.M.; Chinn, L.W.; Gow, J.M.; Kroetz, D.L.; Klein, T.E.; Altman, R.B. Very important pharmacogene summary: ABCB1 (MDR1, P-glycoprotein). Pharm. Genom. 2011, 21, 152-161. [CrossRef]

32. Mao, Q.; Unadkat, J.D. Role of the breast cancer resistance protein (BCRP/ABCG2) in drug transport-an update. AAPS J. 2015, 17, 65-82. [CrossRef] 
33. Pfeifer, N.D.; Hardwick, R.N.; Brouwer, K.L. Role of hepatic efflux transporters in regulating systemic and hepatocyte exposure to xenobiotics. Annu. Rev. Pharmacol. Toxicol. 2014, 54, 509-535. [CrossRef] [PubMed]

34. Kellick, K.A.; Bottorff, M.; Toth, P.P. The National Lipid Association's Safety Task F. A clinician's guide to statin drug-drug interactions. J. Clin. Lipidol. 2014, 8, S30-S46. [CrossRef] [PubMed]

35. Keskitalo, J.E.; Zolk, O.; Fromm, M.F.; Kurkinen, K.J.; Neuvonen, P.J.; Niemi, M. ABCG2 polymorphism markedly affects the pharmacokinetics of atorvastatin and rosuvastatin. Clin. Pharmacol. Ther. 2009, 86, 197-203. [CrossRef] [PubMed]

36. Elsby, R.; Hilgendorf, C.; Fenner, K. Understanding the critical disposition pathways of statins to assess drug-drug interaction risk during drug development: It's not just about OATP1B1. Clin. Pharmacol. Ther. 2012, 92, 584-598. [CrossRef]

37. Shirasaka, Y.; Sager, J.E.; Lutz, J.D.; Davis, C.; Isoherranen, N. Inhibition of CYP2C19 and CYP3A4 by omeprazole metabolites and their contribution to drug-drug interactions. Drug Metab. Dispos. 2013, 41, 1414-1424. [CrossRef]

38. Stolbach, A.; Paziana, K.; Heverling, H.; Pham, P. A review of the toxicity of HIV medications II: Interactions with drugs and complementary and alternative medicine products. J. Med. Toxicol. 2015, 11, 326-341. [CrossRef] [PubMed]

39. Bottiger, Y.; Tybring, G.; Gotharson, E.; Bertilsson, L. Inhibition of the sulfoxidation of omeprazole by ketoconazole in poor and extensive metabolizers of S-mephenytoin. Clin. Pharmacol. Ther. 1997, 62, 384-391. [CrossRef]

40. May, M.; Schindler, C. Clinically and pharmacologically relevant interactions of antidiabetic drugs. Ther. Adv. Endocrinol. Metab. 2016, 7, 69-83. [CrossRef]

41. Wiebe, S.T.; Giessmann, T.; Hohl, K.; Schmidt-Gerets, S.; Hauel, E.; Jambrecina, A.; Bader, K.; Ishiguro, N.; Taub, M.E.; Sharma, A.; et al. Validation of a drug transporter probe cocktail using the prototypical inhibitors rifampin, probenecid, verapamil, and cimetidine. Clin. Pharm. 2020, 59, 1627-1639. [CrossRef] [PubMed]

42. Graham, G.G.; Punt, J.; Arora, M.; Day, R.O.; Doogue, M.P.; Duong, J.K.; Furlong, T.J.; Greenfield, J.R.; Greenup, L.C.; Kirkpatrick, C.M.; et al. Clinical pharmacokinetics of metformin. Clin. Pharm. 2011, 50, 81-98. [CrossRef] [PubMed]

43. Moody, D.E.; Fang, W.B.; Lin, S.N.; Weyant, D.M.; Strom, S.C.; Omiecinski, C.J. Effect of rifampin and nelfinavir on the metabolism of methadone and buprenorphine in primary cultures of human hepatocytes. Drug Metab. Dispos. 2009, 37, 2323-2329. [CrossRef]

44. Coffman, B.L.; King, C.D.; Rios, G.R.; Tephly, T.R. The glucuronidation of opioids, other xenobiotics, and androgens by human UGT2B7Y(268) and UGT2B7H(268). Drug Metab. Dispos. 1998, 26, 73-77.

45. Cheng, Z.; Rios, G.R.; King, C.D.; Coffman, B.L.; Green, M.D.; Mojarrabi, B.; Mackenzie, P.I.; Tephly, T.R. Glucuronidation of catechol estrogens by expressed human UDP-glucuronosyltransferases (UGTs) 1A1, 1A3, and 2B7. Toxicol. Sci. 1998, $45,52-57$. [CrossRef]

46. Papathanasiou, T.; Springborg, A.D.; Kongstad, K.T.; Staerk, D.; Moller, K.; Taylor, B.K.; Lund, T.M.; Werner, M.U. High-dose naloxone, an experimental tool uncovering latent sensitisation: Pharmacokinetics in humans. Br. J. Anaesth. 2019, 123, e204-e214. [CrossRef]

47. Jiang, X.L.; Samant, S.; Lesko, L.J.; Schmidt, S. Clinical pharmacokinetics and pharmacodynamics of clopidogrel. Clin. Pharm. 2015, 54, 147-166. [CrossRef]

48. Mistry, S.D.; Trivedi, H.R.; Parmar, D.M.; Dalvi, P.S.; Jiyo, C. Impact of proton pump inhibitors on efficacy of clopidogrel: Review of evidence. Indian J. Pharmacol. 2011, 43, 183-186. [CrossRef]

49. Bonello, L.; Tantry, U.S.; Marcucci, R.; Blindt, R.; Angiolillo, D.J.; Becker, R.; Bhatt, D.L.; Cattaneo, M.; Collet, J.P.; Cuisset, T.; et al. Consensus and future directions on the definition of high on-treatment platelet reactivity to adenosine diphosphate. J. Am. Coll Cardiol. 2010, 56, 919-933. [CrossRef] [PubMed]

50. Venkatakrishnan, K.; Greenblatt, D.J.; von Moltke, L.L.; Shader, R.I. Alprazolam is another substrate for human cytochrome P450-3A isoforms. J. Clin. Psychopharmacol. 1998, 18, 256. [CrossRef] [PubMed]

51. Hakkola, J.; Hukkanen, J.; Turpeinen, M.; Pelkonen, O. Inhibition and induction of CYP enzymes in humans: An update. Arch. Toxicol. 2020, 94, 3671-3722. [CrossRef]

52. Baumann, P. Pharmacology and pharmacokinetics of citalopram and other SSRIs. Int. Clin. Psychopharmacol. 1996, 11 (Suppl. 1), 5-11. [CrossRef] [PubMed]

53. Kobayashi, K.; Chiba, K.; Yagi, T.; Shimada, N.; Taniguchi, T.; Horie, T.; Tani, M.; Yamamoto, T.; Ishizaki, T.; Kuroiwa, Y. Identification of cytochrome P450 isoforms involved in citalopram $\mathrm{N}$-demethylation by human liver microsomes. J. Pharmacol. Exp. Ther. 1997, 280, 927-933. [PubMed]

54. Sanchez, R.I.; Fillgrove, K.L.; Yee, K.L.; Liang, Y.; Lu, B.; Tatavarti, A.; Liu, R.; Anderson, M.S.; Behm, M.O.; Fan, L.; et al. Characterisation of the absorption, distribution, metabolism, excretion and mass balance of doravirine, a non-nucleoside reverse transcriptase inhibitor in humans. Xenobiotica 2019, 49, 422-432. [CrossRef]

55. Bleasby, K.; Fillgrove, K.L.; Houle, R.; Lu, B.; Palamanda, J.; Newton, D.J.; Lin, M.; Chan, G.H.; Sanchez, R.I. In vitro evaluation of the drug interaction potential of doravirine. Antimicrob. Agents Chemother. 2019, 63, e02492-18. [CrossRef] [PubMed]

56. Fahmi, O.A.; Shebley, M.; Palamanda, J.; Sinz, M.W.; Ramsden, D.; Einolf, H.J.; Chen, L.; Wang, H. Evaluation of CYP2B6 induction and prediction of clinical drug-drug interactions: Considerations from the IQ Consortium Induction Working Group-an industry perspective. Drug Metab. Dispos. 2016, 44, 1720-1730. [CrossRef]

57. Chu, X.; Cai, X.; Cui, D.; Tang, C.; Ghosal, A.; Chan, G.; Green, M.; Kuo, Y.; Liang, Y.; Maciolek, C.; et al. In vitro assessment of drug-drug interaction potential of boceprevir associated with drug metabolizing enzymes and transporters. Drug Metab. Dispos. 2013, 41, 668-681. [CrossRef] [PubMed] 
58. Rizk, M.; Houle, R.; Chan, G.; Hafey, M.; Rhee, E.; Chu, X. Raltegravir has a low propensity to cause clinical drug interactions through inhibition of major drug transporters: An in vitro evaluation. Antimicrob. Agents Chemother. 2014, 58, $1294-1301$. [CrossRef]

59. Chu, X.; Bleasby, K.; Yabut, J.; Cai, X.; Chan, G.; Hafey, M.; Xu, S.; Bergman, A.; Braun, M.; Dean, D.; et al. Transport of the dipeptidyl peptidase-4 inhibitor sitagliptin by human organic anion transporter 3, organic anion transporting polypeptide $4 \mathrm{C} 1$, and multidrug resistance P-glycoprotein. J. Pharmacol. Exp. Ther. 2007, 321, 673-683. [CrossRef]

60. The Human Protein Atlas. ADA. 2020. Available online: https://www.proteinatlas.org/ENSG00000196839-ADA/tissue (accessed on 22 July 2021).

61. Kirby, K.A.; Michailidis, E.; Fetterly, T.L.; Steinbach, M.A.; Singh, K.; Marchand, B.; Leslie, M.D.; Hagedorn, A.N.; Kodama, E.N.; Marquez, V.E.; et al. Effects of substitutions at the $4^{\prime}$ and 2 positions on the bioactivity of $4^{\prime}$-ethynyl-2-fluoro- $2^{\prime}$-deoxyadenosine. Antimicrob. Agents Chemother. 2013, 57, 6254-6264. [CrossRef]

62. Austin, R.; Barton, P.; Cockroft, S.; Wenlock, M.; Riley, R. The influence of nonspecific microsomal binding on apparent intrinsic clearance, and its prediction from physicochemical properties. Drug Metab. Dispos. 2002, 30, 1497-1503. [CrossRef] [PubMed]

63. Tanaka, E. Clinically important pharmacokinetic drug-drug interactions: Role of cytochrome P450 enzymes. J. Clin. Pharm. Ther. 1998, 23, 403-416. [CrossRef]

64. Zanger, U.M.; Schwab, M. Cytochrome P450 enzymes in drug metabolism: Regulation of gene expression, enzyme activities, and impact of genetic variation. Pharmacol. Ther. 2013, 138, 103-141. [CrossRef] [PubMed]

65. Low, Y.; Setia, S.; Lima, G. Drug-drug interactions involving antidepressants: Focus on desvenlafaxine. Neuropsychiatr. Dis. Treat. 2018, 14, 567-580. [CrossRef] [PubMed]

66. McCance-Katz, E.F.; Sullivan, L.E.; Nallani, S. Drug interactions of clinical importance among the opioids, methadone and buprenorphine, and other frequently prescribed medications: A review. Am. J. Addict. 2010, 19, 4-16. [CrossRef] [PubMed]

67. Lv, H.H.; Wu, S.; Liu, X.; Yang, X.L.; Xu, J.F.; Guan, Y.T.; Dong, Q.; Zheng, S.L.; Jiang, J.M.; Li, S.X.; et al. Comparison of VerifyNow P2Y12 and thrombelastography for assessing clopidogrel response in stroke patients in China. Neurol. Sci. 2016, 37, $277-282$. [CrossRef]

68. Zhou, S.F.; Wang, L.L.; Di, Y.M.; Xue, C.C.; Duan, W.; Li, C.G.; Li, Y. Substrates and inhibitors of human multidrug resistance associated proteins and the implications in drug development. Curr. Med. Chem. 2008, 15, 1981-2039. [CrossRef]

69. Kenna, J.; Taskar, K.; Battista, C.; Bourdet, D.; Brouwer, K.; Brouwer, K.; Dai, D.; Funk, C.; Hafey, M.; Lai, Y.; et al. Can bile salt export pump inhibition testing in drug discovery and development reduce liver injury risk? an international transporter consortium perspective. Clin. Pharm. Ther. 2018, 104, 916-932. [CrossRef]

70. Lepist, E.I.; Ray, A.S. Renal transporter-mediated drug-drug interactions: Are they clinically relevant? J. Clin. Pharmacol. 2016, 56 (Suppl. 7), S73-S81. [CrossRef] [PubMed]

71. Kearney, B.P.; Flaherty, J.F.; Shah, J. Tenofovir disoproxil fumarate: Clinical pharmacology and pharmacokinetics. Clin. Pharm. 2004, 43, 595-612. [CrossRef]

72. Fernandez-Fernandez, B.; Montoya-Ferrer, A.; Sanz, A.B.; Sanchez-Nino, M.D.; Izquierdo, M.C.; Poveda, J.; Sainz-Prestel, V.; Ortiz-Martin, N.; Parra-Rodriguez, A.; Selgas, R.; et al. Tenofovir nephrotoxicity: 2011 update. AIDS Res. Treat. 2011, 2011, 354908. [CrossRef] [PubMed]

73. Jackson Rudd, D.; Cao, Y.; Vaddady, P.; Grobler, J.A.; Asante-Appiah, E.; Diamond, T.; Klopfer, S.; Grandhi, A.; Hwang, C.; Vargo, R. Modeling-Supported Islatravir Dose Selection for Phase 3. In Proceedings of the Conference on Retroviruses and Opportunistic Infections (CROI), Boston, MA, USA, 8-11 March 2020.

74. Matthews, R.; Ankrom, W.; Friedman, E.; Jackson Rudd, D.; Liu, Y.; Mogg, R.; Panebianco, D.; De Lepeleire, I.; Petkova, M.; Grobler, J.; et al. Safety, tolerability, and pharmacokinetics of single- and multiple-dose administration of islatravir (MK-8591) in adults without HIV. Clin. Trans. Sci. 2021, in press.

75. Matthews, R.; Jackson Rudd, D.; Fillgrove, K.; Zhang, S.; Tomek, C.; Stoch, S.A.; Iwamoto, M. A phase 1 study to evaluate the drug interaction between islatravir (MK-8591) and doravirine in adults without HIV. Clin. Drug Investig. 2021, in press. [CrossRef]

76. Reese, M.J.; Savina, P.M.; Generaux, G.T.; Tracey, H.; Humphreys, J.E.; Kanaoka, E.; Webster, L.O.; Harmon, K.A.; Clarke, J.D.; Polli, J.W. In vitro investigations into the roles of drug transporters and metabolizing enzymes in the disposition and drug interactions of dolutegravir, a HIV integrase inhibitor. Drug Metab. Dispos. 2013, 41, 353-361. [CrossRef] [PubMed]

77. Ankrom, W.; Jonathan, D.; Jackson Rudd, D.; Zhang, S.; Gravesande, K.; Matthews, R.; Brimhall, D.; Stoch, S.A.; Iwamoto, M. MK-8591 Does Not Alter the Pharmacokinetics of the Oral Contraceptives Levonorgestrel and Ethinyl Estradiol. In Proceedings of the IDWeek 2018, San Francisco, CA, USA, 3-7 October 2018.

78. Pfizer Inc. ALESSE®[Product Information]; Pfizer Canada Inc.: Kirkland, QC, Canada, 2017.

79. US Food and Drug Administration Center for Drug Evaluation and Research. In Vitro Metabolism- and Transporter-Mediated Drug-Drug Interaction Studies Guidance for Industry. 2017. Available online: https://www.fda.gov/media/108130/download (accessed on 22 July 2021). 